\title{
Spurious and true eigensolutions of Helmholtz BIEs and BEMs for a multiply connected problem
}

\author{
By J. T. CHEN ${ }^{1}$, L. W. LIU AND H.-K. HONG ${ }^{2}$ \\ ${ }^{1}$ Department of Harbor and River Engineering, \\ National Taiwan Ocean University, Keelung, \\ Taiwan (jtchen@mail.ntou.edu.tw) \\ ${ }^{2}$ Department of Civil Engineering, National Taiwan University, \\ Taipei, Taiwan
}

Received 21 March 2002; accepted 12 October 2002; published online 5 June 2003

The spurious eigenvalues of an annular domain have been verified for the singular and hypersingular boundary-element methods (BEMs) and circumvented by using the Burton-Miller approach. Do they also occur in other formulations: continuous formulations such as the singular and hypersingular boundary integral equations (BIEs), the null-field BIEs and the fictitious BIEs, or such discrete formulations as the null-field BEMs and the fictitious BEMs? For the ten formulations of the multiply connected problem the study of otherwise the same issues is continued in the present paper. By using the degenerate kernels and the Fourier series, it is demonstrated analytically for the six continuous formulations of BIEs that spurious eigensolutions depend on the geometry of the inner boundary but not on that of the outer boundary. This conclusion can be extended to the six discrete formulations of BEMs. To filter out the spurious eigenvalues, the CHIEF (combined Helmholtz integral equation formulation) method is used here instead of the Burton-Miller approach. The optimum number and appropriate positions of the CHIEF points are also addressed. It is then shown that, in the null-field and fictitious BEMs, the spurious and true eigenvalues can be detected and distinguished by using the singular-value-decompositionupdating techniques in conjunction with the Fredholm alternative theorem. Illustrative examples show the validity of the proposed methodologies.

Keywords: boundary integral equation; Helmholtz equation; spurious eigenvalue; degenerate kernel; Fourier series; multiply connected domain

\section{Introduction}

For the acoustic problem, it is well known that the boundary integral equations (BIEs) for solving the exterior and interior problems result in fictitious frequencies and spurious eigenvalues, respectively. In the exterior acoustics, to deal with the fictitious frequency problem, the Burton-Miller method (Burton \& Miller 1971) and the combined Helmholtz interior integral equation formulation (CHIEF) method (Schenck 1968; Seybert \& Rengarajan 1987) have been proposed. The Burton-Miller method added the hypersingular $(L M)$ equation times the imaginary unit $\mathrm{i}$ to the 
singular $(U T)$ equation, while the CHIEF method imposed additional constraints by collocating the $U T$ equation at points in the complementary domain. Besides, Achenbach et al. (1988) employed an off-boundary approach to avoid fictitious frequencies as well as singularities. Mathematically speaking, the off-boundary approach originates from the null-field formulation (Martin 1980). Another way to avoid singularities is the retracted boundary approach employed by Hwang \& Chang (1991), which is a kind of fictitious BEM based on the indirect method.

In the interior eigenproblem of a simply connected domain, the dual reciprocity method (DRM) (Partridge et al. 1992) and the multiple reciprocity method (MRM) (Kamiya \& Andoh 1993; Nowak \& Brebbia 1989; Nowak \& Neves 1994) have been widely used. One advantage of the MRM, which uses the Laplace-type fundamental solution, is that only real-valued computation is needed (Itagaki \& Brebbia 1993, 1994; Itagaki et al. 1997). Therefore, the MRM is indeed no more than the real part of the complex-valued formulation (Kamiya et al. 1996). Tai \& Shaw (1974) and De Mey (1976, 1977) employed a simplified method of using either only the real-part or only the imaginary-part kernels. Hutchinson $(1985,1988,1991)$ also employed the realpart kernels to solve membrane-vibration problems. However, regardless of whether a real-part singular or hypersingular equation is used, spurious eigenvalues always occur. To deal with the spurious-eigenvalue problem, the dual MRM (Chen et al. 2000a), the real-part dual BEM (Kuo et al. 2000a), the singular-value-decomposition (SVD) updating terms (Golub \& Van Loan 1989; Berry et al. 1999; Chen et al. 2000b) and the generalized singular value decomposition (GSVD) (Kuo et al. 2000b) have been constructed. Also, Chen et al. (2001a) extended the CHIEF concept to the combined Helmholtz exterior integral equation formulation (CHEEF) method for filtering out the spurious eigenvalues. In fact, there are no spurious eigenvalues if the complex-valued BEM is employed for a simply connected problem. Tai \& Shaw (1974) claimed that spurious eigenvalues were not present if the complex-valued kernels were employed. However, all the above issues are true only for the cases of problems with simply connected domains. In a multiply connected problem, spurious eigensolutions always appear, even when the complex-valued BEM is employed to solve the eigensolutions (Kitahara 1985; Chen et al. 2001c). In Chen et al. (2001c), the problem of spurious eigensolutions of the singular and hypersingular BEMs was studied by using circulants for an annular case and treated by using the BurtonMiller approach in a discrete system.

In this paper we not only intend to examine the mechanisms that cause spurious eigensolutions, but also intend to further examine whether the mechanisms vary with different mathematical formulations and numerical methods employed. Sections 2 and 3 solve the complex-valued BIEs for the eigensolutions of an annular domain as a continuous formulation and study analytically the occurring mechanisms of the spurious eigensolutions. The degenerate kernels for the fundamental solution and the Fourier series expansions of boundary densities are employed to find and distinguish analytically the spurious and true eigenvalues for the multiply connected problem. Sections 4 and 5 show that, even being able to avoid singularity, the null-field BIEs and the fictitious BIEs still yield spurious eigensolutions, and that their occurring mechanisms are essentially the same as those of the BIEs. Section 6 uses the CHIEF method to circumvent the problems of spurious eigenvalues for the three continuous formulations of BIEs, also addressing how to choose the positions and determine the number of the CHIEF points. The BEM implementations of the singular and 
hypersingular BIEs, the null-field BIEs and the fictitious BIEs are presented in $\S 7$, where spurious and true eigenvalues are detected and distinguished by using the SVD-updating techniques in conjunction with the Fredholm alternative theorem. Section 8 gives numerical examples and $\S 9$ concludes the paper.

\section{Boundary integral equations of spurious and true eigenvalues for a multiply connected problem}

For the acoustic problem, the governing equation is the Helmholtz equation

$$
\left(\nabla^{2}+k^{2}\right) u(\boldsymbol{x})=0, \quad \boldsymbol{x} \in D
$$

where $\nabla^{2}$ is the Laplace operator, $k$ the wavenumber and $D$ the domain of interest. On the basis of the dual boundary integral formulation (Hong \& Chen 1988; Chen \& Hong 1999), we have

$$
\begin{aligned}
& \alpha u(\boldsymbol{x})=\mathrm{CPV} \int_{B} T(\boldsymbol{s}, \boldsymbol{x}) u(\boldsymbol{s}) \mathrm{d} B(\boldsymbol{s})-\mathrm{RPV} \int_{B} U(\boldsymbol{s}, \boldsymbol{x}) t(\boldsymbol{s}) \mathrm{d} B(\boldsymbol{s}), \quad \boldsymbol{x} \in B, \\
& \alpha t(\boldsymbol{x})=\operatorname{HPV} \int_{B} M(\boldsymbol{s}, \boldsymbol{x}) u(\boldsymbol{s}) \mathrm{d} B(\boldsymbol{s})-\mathrm{CPV} \int_{B} L(\boldsymbol{s}, \boldsymbol{x}) t(\boldsymbol{s}) \mathrm{d} B(\boldsymbol{s}), \quad \boldsymbol{x} \in B,
\end{aligned}
$$

where $\boldsymbol{x}$ is the field point, $s$ the source point, $B$ the boundary, RPV the Riemann principal value, CPV the Cauchy principal value, HPV the Hadamard principal value, $t(\boldsymbol{s})$ the directional derivative of $u(\boldsymbol{s})$ along the outer normal direction at $\boldsymbol{s}$ and $\alpha$ the interior angle of the boundary at $\boldsymbol{x}$. The $U(\boldsymbol{s}, \boldsymbol{x}), T(\boldsymbol{s}, \boldsymbol{x}), L(\boldsymbol{s}, \boldsymbol{x})$ and $M(\boldsymbol{s}, \boldsymbol{x})$ represent the four kernel functions (Chen \& Chen 1998),

$$
\begin{aligned}
U(\boldsymbol{s}, \boldsymbol{x}) & =-\frac{1}{2} \mathrm{i} \pi H_{0}^{(1)}(k r), \\
T(\boldsymbol{s}, \boldsymbol{x}) & =-\frac{1}{2} \mathrm{i} k \pi H_{1}^{(1)}(k r) \frac{y_{i} n_{i}}{r}, \\
L(\boldsymbol{s}, \boldsymbol{x}) & =\frac{1}{2} \mathrm{i} k \pi H_{1}^{(1)}(k r) \frac{y_{i} \bar{n}_{i}}{r}, \\
M(\boldsymbol{s}, \boldsymbol{x}) & =-\frac{1}{2} \mathrm{i} k \pi\left[-k \frac{H_{2}^{(1)}(k r)}{r^{2}} y_{i} y_{j} n_{i} \bar{n}_{j}+\frac{H_{1}^{1}(k r)}{r} n_{i} \bar{n}_{i}\right],
\end{aligned}
$$

where $H_{n}^{(1)}(k r)$ is the $n$ th-order Hankel function of the first kind, $r=|\boldsymbol{x}-\boldsymbol{s}|$, $y_{i}=s_{i}-x_{i}, \mathrm{i}^{2}=-1$ and $n_{i}$ and $\bar{n}_{i}$ are the $i$ th components of the outer normal vectors at $\boldsymbol{s}$ and $\boldsymbol{x}$, respectively. Equation (2.2) is referred to as the singular BIE, equation (2.3) the hypersingular BIE. The combined use of both is coined the dual BIEs. Corresponding to (2.2) and (2.3), the null-field BIEs (Martin 1980) based on the direct method are

$$
\begin{array}{ll}
0=\int_{B} T(\boldsymbol{s}, \boldsymbol{x}) u(\boldsymbol{s}) \mathrm{d} B(\boldsymbol{s})-\int_{B} U(\boldsymbol{s}, \boldsymbol{x}) t(\boldsymbol{s}) \mathrm{d} B(\boldsymbol{s}), & \boldsymbol{x} \in D^{\mathrm{e}}, \\
0=\int_{B} M(\boldsymbol{s}, \boldsymbol{x}) u(\boldsymbol{s}) \mathrm{d} B(\boldsymbol{s})-\int_{B} L(\boldsymbol{s}, \boldsymbol{x}) t(\boldsymbol{s}) \mathrm{d} B(\boldsymbol{s}), & \boldsymbol{x} \in D^{\mathrm{e}},
\end{array}
$$

where $D^{\mathrm{e}}$ is the complementary domain and the kernels are the same as listed in (2.4)-(2.7). Note that the null-field BIEs are not singular. 


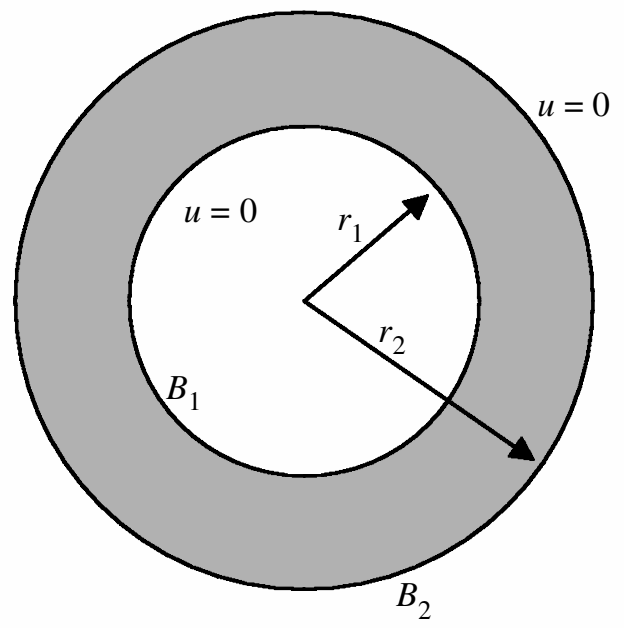

Figure 1. Sketch of an annular cavity.
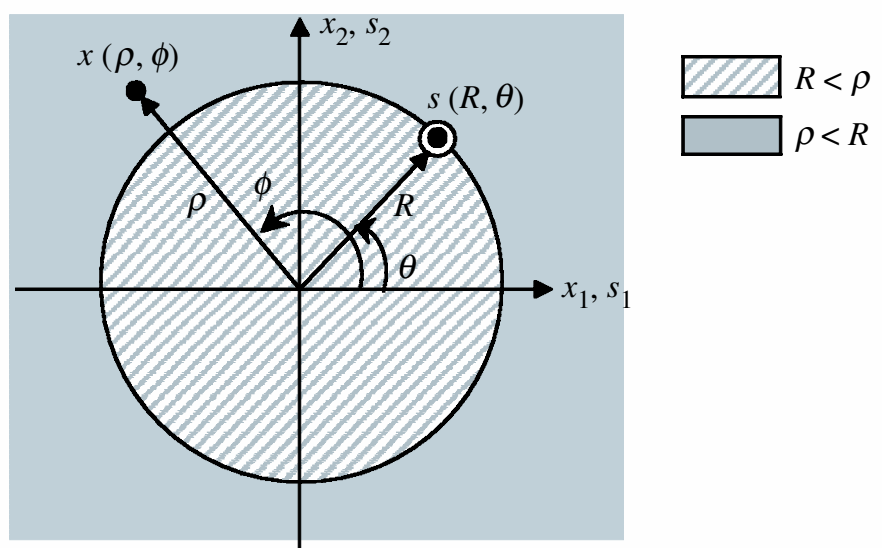

Figure 2. Symbols for the degenerate kernels.

For the purposes of analytical study, we consider an annular case and adopt such analytical tools as degenerate kernels and Fourier series. Figure 1 shows an annular cavity with boundary $B$ containing the inner and outer boundaries, $B_{1}$ and $B_{2}$, the radii of which are $r_{1}$ and $r_{2}$, respectively. To prepare for the annular case, let us consider beforehand a circular case, as shown in figure 2, for which the kernel functions (2.4)-(2.7) in the null-field BIEs (2.8) and (2.9) can be expanded into the following degenerate kernels,

$$
U(\boldsymbol{s}, \boldsymbol{x})=\left\{\begin{aligned}
U^{\mathrm{i}}(\boldsymbol{s}, \boldsymbol{x}) & =\sum_{m=-\infty}^{\infty} \frac{1}{2} \pi J_{m}(k \rho)\left(Y_{m}(k R)-\mathrm{i} J_{m}(k R)\right) \cos (m(\theta-\phi)), \\
R & >\rho, \\
U^{\mathrm{e}}(\boldsymbol{s}, \boldsymbol{x}) & =\sum_{m=-\infty}^{\infty} \frac{1}{2} \pi J_{m}(k R)\left(Y_{m}(k \rho)-\mathrm{i} J_{m}(k \rho)\right) \cos (m(\theta-\phi)), \\
\rho & >R,
\end{aligned}\right.
$$




$$
\begin{aligned}
& T(\boldsymbol{s}, \boldsymbol{x})=\left\{\begin{array}{rr}
T^{\mathrm{i}}(\boldsymbol{s}, \boldsymbol{x})=\sum_{m=-\infty}^{\infty} \frac{1}{2} \pi k J_{m}(k \rho)\left(Y_{m}^{\prime}(k R)-\mathrm{i} J_{m}^{\prime}(k R)\right) \cos (m(\theta-\phi)), & R>\rho, \\
T^{\mathrm{e}}(\boldsymbol{s}, \boldsymbol{x})=\sum_{m=-\infty}^{\infty} \frac{1}{2} \pi k J_{m}^{\prime}(k R)\left(Y_{m}(k \rho)-\mathrm{i} J_{m}(k \rho)\right) \cos (m(\theta-\phi)), & \rho>R,
\end{array}\right. \\
& L(\boldsymbol{s}, \boldsymbol{x})=\left\{\begin{aligned}
L^{\mathrm{i}}(\boldsymbol{s}, \boldsymbol{x})= & \sum_{m=-\infty}^{\infty} \frac{1}{2} \pi k J_{m}^{\prime}(k \rho)\left(Y_{m}(k R)-\mathrm{i} J_{m}(k R)\right) \cos (m(\theta-\phi)), \\
L^{\mathrm{e}}(\boldsymbol{s}, \boldsymbol{x})=\sum_{m=-\infty}^{\infty} \frac{1}{2} \pi k J_{m}(k R)\left(Y_{m}^{\prime}(k \rho)-\mathrm{i} J_{m}^{\prime}(k \rho)\right) \cos (m(\theta-\phi)), & \rho>R,
\end{aligned}\right. \\
& M(\boldsymbol{s}, \boldsymbol{x})=\left\{\begin{array}{r}
M^{\mathrm{i}}(\boldsymbol{s}, \boldsymbol{x})=\sum_{m=-\infty}^{\infty} \frac{1}{2} \pi k^{2} J_{m}^{\prime}(k \rho)\left(Y_{m}^{\prime}(k R)-\mathrm{i} J_{m}^{\prime}(k R)\right) \cos (m(\theta-\phi)), \\
R>\rho, \\
M^{\mathrm{e}}(\boldsymbol{s}, \boldsymbol{x})=\sum_{m=-\infty}^{\infty} \frac{1}{2} \pi k^{2} J_{m}^{\prime}(k R)\left(Y_{m}^{\prime}(k \rho)-\mathrm{i} J_{m}^{\prime}(k \rho)\right) \cos (m(\theta-\phi)), \\
\rho>R,
\end{array}\right.
\end{aligned}
$$

where $(R, \theta)$ and $(\rho, \phi)$ are the polar coordinates of $\boldsymbol{s}$ and $\boldsymbol{x}$, respectively. Note that $U=U^{\mathrm{i}}, T=T^{\mathrm{i}}, L=L^{\mathrm{i}}$ and $M=M^{\mathrm{i}}$ for the exterior circular problem and $U=U^{\mathrm{e}}$, $T=T^{\mathrm{e}}, L=L^{\mathrm{e}}$ and $M=M^{\mathrm{e}}$ for the interior circular problem.

With the above preparation, we are now ready for a study of the annular case. If, for simplicity, we further subject the problem to the Dirichlet boundary condition $u=0$ on all $B$, the null-field equation (2.8) simplifies to

$$
\int_{B} U(\boldsymbol{s}, \boldsymbol{x}) t(\boldsymbol{s}) \mathrm{d} B(\boldsymbol{s})=0, \quad \boldsymbol{x} \in D^{\mathrm{e}},
$$

and hence only $t(s)$ on $B_{1}$ and $B_{2}$ need be expanded into the Fourier series,

$$
\begin{array}{ll}
t(\boldsymbol{s})=a_{0}+\sum_{n=1}^{\infty}\left(a_{n} \cos n \theta+b_{n} \sin n \theta\right), & \boldsymbol{s} \in B_{1}, \\
t(\boldsymbol{s}) & =p_{0}+\sum_{n=1}^{\infty}\left(p_{n} \cos n \theta+q_{n} \sin n \theta\right), \quad \boldsymbol{s} \in B_{2},
\end{array}
$$

where $a_{n}, b_{n}, p_{n}$ and $q_{n}$ are the Fourier coefficients. In the stage of substituting (2.10) for the $U$ kernel in $(2.14), U^{\mathrm{i}}$ and $U^{\mathrm{e}}$ are adopted for the field point $\boldsymbol{x}=(\rho, \phi)$ in the inner complementary domain $\left(\rho<r_{1}\right)$ and the outer complementary domain $\left(\rho>r_{2}\right)$, respectively. When the field point $\boldsymbol{x}$ with radius $\rho<r_{1}$ approaches the 
boundary $B_{1}$ and is finally located on $B_{1}$ with radius $\rho=r_{1}$, we have

$$
\begin{aligned}
& \int_{B_{2}} U^{\mathrm{i}}(\boldsymbol{s}, \boldsymbol{x}) t(\boldsymbol{s}) \mathrm{d} B(\boldsymbol{s})+\int_{B_{1}} U^{\mathrm{i}}(\boldsymbol{s}, \boldsymbol{x}) t(\boldsymbol{s}) \mathrm{d} B(\boldsymbol{s}) \\
&= \sum_{n=0}^{\infty} \frac{1}{\varepsilon_{n}} \pi^{2} r_{2} J_{n}\left(k r_{1}\right)\left[Y_{n}\left(k r_{2}\right)-\mathrm{i} J_{n}\left(k r_{2}\right)\right]\left(p_{n} \cos n \phi+q_{n} \sin n \phi\right) \\
&-\sum_{n=0}^{\infty} \frac{1}{\varepsilon_{n}} \pi^{2} r_{1} J_{n}\left(k r_{1}\right)\left[Y_{n}\left(k r_{1}\right)-\mathrm{i} J_{n}\left(k r_{1}\right)\right]\left(a_{n} \cos n \phi+b_{n} \sin n \phi\right)=0, \\
& \boldsymbol{x} \in B_{1},
\end{aligned}
$$

where $\varepsilon_{n}$ is the Neumann factor $\left(\varepsilon_{0}=1\right.$ and $\varepsilon_{n}=2$ for $\left.n>0\right)$. According to (2.17), the Fourier coefficients $a_{n}, p_{n}, b_{n}$ and $q_{n}$ have the following relations:

$$
\begin{aligned}
& p_{n}=\left(\frac{r_{1} J_{n}\left(k r_{1}\right)\left[Y_{n}\left(k r_{1}\right)-\mathrm{i} J_{n}\left(k r_{1}\right)\right]}{r_{2} J_{n}\left(k r_{1}\right)\left[Y_{n}\left(k r_{2}\right)-\mathrm{i} J_{n}\left(k r_{2}\right)\right]}\right) a_{n}, \quad n=0,1,2, \ldots, \\
& q_{n}=\left(\frac{r_{1} J_{n}\left(k r_{1}\right)\left[Y_{n}\left(k r_{1}\right)-\mathrm{i} J_{n}\left(k r_{1}\right)\right]}{r_{2} J_{n}\left(k r_{1}\right)\left[Y_{n}\left(k r_{2}\right)-\mathrm{i} J_{n}\left(k r_{2}\right)\right]}\right) b_{n}, \quad n=1,2, \ldots
\end{aligned}
$$

On the other hand, moving the field point $\boldsymbol{x}$ with radius $\rho>r_{2}$ to the boundary $B_{2}$ and finally locating it on $B_{2}$ with radius $\rho=r_{2}$, we have

$$
\begin{aligned}
& \int_{B_{2}} U^{\mathrm{e}}(\boldsymbol{s}, \boldsymbol{x}) t(\boldsymbol{s}) \mathrm{d} B(\boldsymbol{s})+\int_{B_{1}} U^{\mathrm{e}}(\boldsymbol{s}, \boldsymbol{x}) t(\boldsymbol{s}) \mathrm{d} B(\boldsymbol{s}) \\
&= \sum_{n=0}^{\infty} \frac{1}{\varepsilon_{n}} \pi^{2} r_{2} J_{n}\left(k r_{2}\right)\left[Y_{n}\left(k r_{2}\right)-\mathrm{i} J_{n}\left(k r_{2}\right)\right]\left(p_{n} \cos n \phi+q_{n} \sin n \phi\right) \\
&-\sum_{n=0}^{\infty} \frac{1}{\varepsilon_{n}} \pi^{2} r_{1} J_{n}\left(k r_{1}\right)\left[Y_{n}\left(k r_{2}\right)-\mathrm{i} J_{n}\left(k r_{2}\right)\right]\left(a_{n} \cos n \phi+b_{n} \sin n \phi\right)=0, \\
& \boldsymbol{x} \in B_{2} .
\end{aligned}
$$

Similarly, we obtain the relations between $a_{n}, b_{n}$ and $p_{n}, q_{n}$ as follows:

$$
\begin{aligned}
& p_{n}=\left(\frac{r_{1} J_{n}\left(k r_{1}\right)\left[Y_{n}\left(k r_{2}\right)-\mathrm{i} J_{n}\left(k r_{2}\right)\right]}{r_{2} J_{n}\left(k r_{2}\right)\left[Y_{n}\left(k r_{2}\right)-\mathrm{i} J_{n}\left(k r_{2}\right)\right]}\right) a_{n}, \quad n=0,1,2, \ldots, \\
& q_{n}=\left(\frac{r_{1} J_{n}\left(k r_{1}\right)\left[Y_{n}\left(k r_{2}\right)-\mathrm{i} J_{n}\left(k r_{2}\right)\right]}{r_{2} J_{n}\left(k r_{2}\right)\left[Y_{n}\left(k r_{2}\right)-\mathrm{i} J_{n}\left(k r_{2}\right)\right]}\right) b_{n}, \quad n=1,2, \ldots
\end{aligned}
$$

To seek non-trivial data for the generalized coefficients of $a_{n}, b_{n}, p_{n}$ and $q_{n}$, we have two possible eigensolutions for (2.18), (2.19), (2.21) and (2.22). Firstly, we have the eigenequations

$$
J_{n}\left(k r_{1}\right) Y_{n}\left(k r_{2}\right)-J_{n}\left(k r_{2}\right) Y_{n}\left(k r_{1}\right)=0, \quad n=0,1,2, \ldots
$$


from which we obtain the non-trivial data in column vector form:

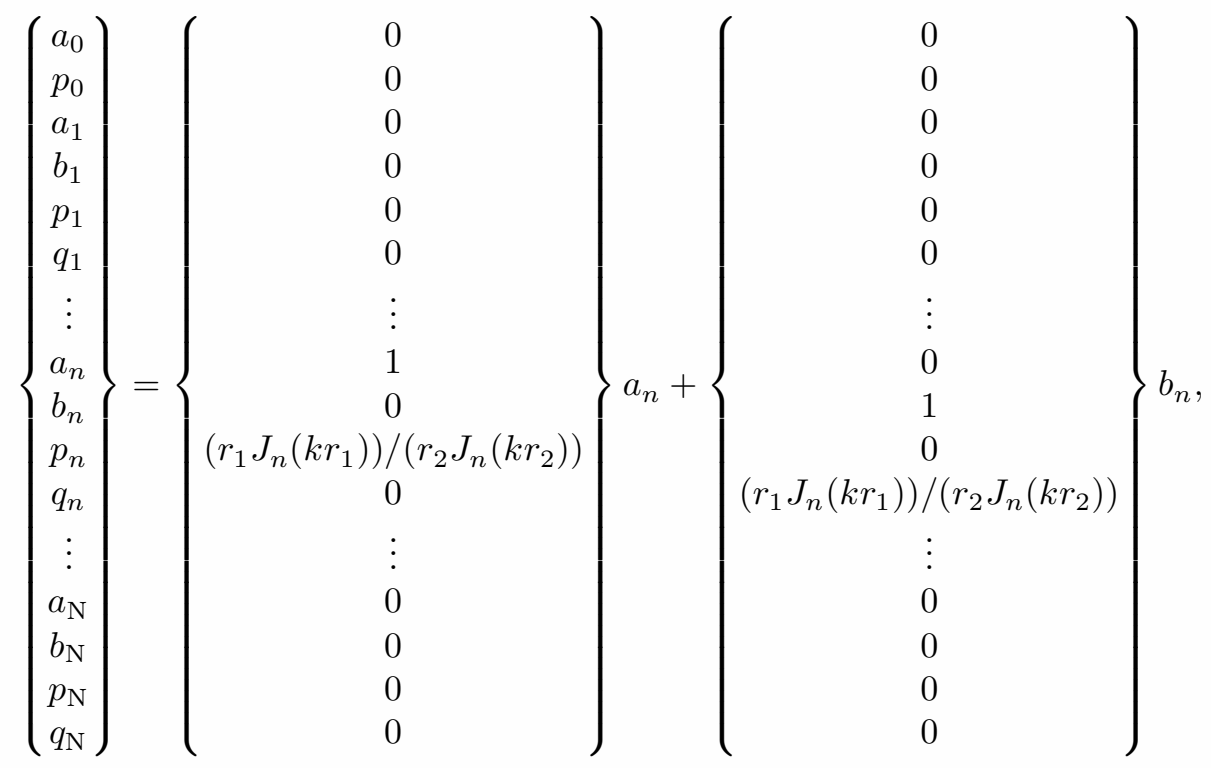

where $a_{n}$ and $b_{n}$ are arbitrary. Secondly, we have

$$
J_{n}\left(k r_{1}\right)=0, \quad n=0,1,2, \ldots,
$$

from which we obtain the non-trivial data

$$
\left\{\begin{array}{c}
a_{0} \\
p_{0} \\
a_{1} \\
b_{1} \\
p_{1} \\
q_{1} \\
\vdots \\
a_{n} \\
b_{n} \\
p_{n} \\
q_{n} \\
\vdots \\
a_{\mathrm{N}} \\
b_{\mathrm{N}} \\
p_{\mathrm{N}} \\
q_{\mathrm{N}}
\end{array}\right\}=\left\{\begin{array}{c}
0 \\
0 \\
0 \\
0 \\
0 \\
0 \\
\vdots \\
1 \\
0 \\
0 \\
0 \\
\vdots \\
0 \\
0 \\
0 \\
0
\end{array}\right\} a_{n}+\left\{\begin{array}{c}
0 \\
0 \\
0 \\
0 \\
0 \\
0 \\
\vdots \\
1 \\
0 \\
0 \\
\vdots \\
0 \\
0 \\
0 \\
0
\end{array}\right\} b_{n}
$$

where $a_{n}$ and $b_{n}$ are also arbitrary.

Similarly, we can derive two possible eigenequations if the hypersingular $(L M)$ BIE is used. The first is identical to (2.23), but the second now turns out to be

$$
J_{n}^{\prime}\left(k r_{1}\right)=0, \quad n=0,1,2, \ldots,
$$


from which we also obtain the data (2.26). In (2.27), the prime denotes the derivative with respect to the argument.

It is interesting and critically important to note that, although $J_{n}$ of $(2.25)$ and $J_{n}^{\prime}$ of (2.27) have different sets of roots, their corresponding non-trivial data for the generalized coefficients are the same, that is, those corresponding to (2.27) are identical to the data (2.26). Let us recall, however, that (2.23) is the same set of eigenequations resulted from either using the singular $(U T)$ BIE or the hypersingular $(L M)$ BIE. This indicates that (2.23) is the true set of eigenequations and the true eigensolutions are indeed as they are, independent of whatever different formulations being used. Conversely, the spurious eigenvalues that satisfy the spurious eigenequations are instead hiding in (and as a consequence depend upon) the particular formulations used; therefore, equations (2.25) and (2.27) are the spurious eigenequations analytically derived from the singular and hypersingular BIEs, respectively. According to (2.25) (respectively, (2.27)), the spurious eigenvalues $k_{\mathrm{s}}$ hiding in the singular (respectively, hypersingular) BIE are the roots of $J_{n}$ (respectively, $J_{n}^{\prime}$ ) divided by $r_{1}$ and hence depend on the inner boundary $\left(r_{1}\right)$ of the annular domain. This assertion matches the results obtained by using circulants and numerical simulations reported in Chen et al. (2001c). In fact, the multiply connected problem can be superimposed by two problems: one an interior problem with $B_{2}$ as the boundary and the other an exterior problem with $B_{1}$ as the boundary (Chen et al. 2001c). The source that causes the spurious eigenvalues stems from the exterior problem with the inner boundary, since it is well known that fictitious frequencies are embedded in the integral formulation, even though the complex-valued kernels (of the $U T$ or $L M$ equations) are employed (Chen 1998; Chen \& Kuo 2000).

\section{Derivation of true and spurious modes by using degenerate kernels and the Fourier series expansions}

In the last section we have identified the spurious and true eigenvalues. To find the corresponding spurious and true eigenmodes, we first write down the dual BIEs for the interior point $\boldsymbol{x} \in D$ as follows:

$$
\begin{array}{ll}
u(\boldsymbol{x})=-\frac{1}{2 \pi} \int_{B} U(\boldsymbol{s}, \boldsymbol{x}) t(\boldsymbol{s}) \mathrm{d} B(\boldsymbol{s}), & \boldsymbol{x} \in D, \\
t(\boldsymbol{x})=-\frac{1}{2 \pi} \int_{B} L(\boldsymbol{s}, \boldsymbol{x}) t(\boldsymbol{s}) \mathrm{d} B(\boldsymbol{s}), & \boldsymbol{x} \in D,
\end{array}
$$

which, upon using the degenerate kernels (2.4) and (2.6), become

$$
\begin{array}{ll}
u(\boldsymbol{x})=-\frac{1}{2 \pi}\left\{\int_{B_{1}} U^{\mathrm{e}}(\boldsymbol{s}, \boldsymbol{x}) t(\boldsymbol{s}) \mathrm{d} B(\boldsymbol{s})+\int_{B_{2}} U^{\mathrm{i}}(\boldsymbol{s}, \boldsymbol{x}) t(\boldsymbol{s}) \mathrm{d} B(\boldsymbol{s})\right\}, & \boldsymbol{x} \in D, \\
t(\boldsymbol{x})=-\frac{1}{2 \pi}\left\{\int_{B_{1}} L^{\mathrm{e}}(\boldsymbol{s}, \boldsymbol{x}) t(\boldsymbol{s}) \mathrm{d} B(\boldsymbol{s})+\int_{B_{2}} L^{\mathrm{i}}(\boldsymbol{s}, \boldsymbol{x}) t(\boldsymbol{s}) \mathrm{d} B(\boldsymbol{s})\right\}, & \boldsymbol{x} \in D .
\end{array}
$$

Using the boundary data (2.24), we convert the domain equations (3.3) and (3.4) into the true eigenmodes (for $\boldsymbol{x} \in D$ ) as follows:

$$
\begin{gathered}
u_{n}(\rho, \phi)=\frac{\pi}{2 \varepsilon_{n}} r_{1} J_{n}\left(k r_{1}\right)\left[Y_{n}(k \rho)-\frac{Y_{n}\left(k r_{2}\right)}{J_{n}\left(k r_{2}\right)} J_{n}(k \rho)\right]\left(a_{n} \cos n \phi+b_{n} \sin n \phi\right), \\
n=0,1,2, \ldots,
\end{gathered}
$$




$$
t_{n}(\rho, \phi)=\frac{\pi}{2 \varepsilon_{n}} r_{1} k J_{n}\left(k r_{1}\right)\left[Y_{n}^{\prime}(k \rho)-\frac{Y_{n}\left(k r_{2}\right)}{J_{n}\left(k r_{2}\right)} J_{n}^{\prime}(k \rho)\right]\left(a_{n} \cos n \phi+b_{n} \sin n \phi\right),
$$

On the other hand, using the boundary data (2.26), we convert the domain equations (3.3) and (3.4) into the spurious modes (for $\boldsymbol{x} \in D$ ) as follows:

$$
\begin{array}{lr}
u_{n}(\rho, \phi)=\frac{\pi}{2 \varepsilon_{n}} r_{1} J_{n}\left(k r_{1}\right)\left[Y_{n}(k \rho)-\mathrm{i} J_{n}(k \rho)\right]\left(a_{n} \cos n \phi+b_{n} \sin n \phi\right), & n=0,1,2, \ldots, \\
t_{n}(\rho, \phi)=\frac{\pi}{2 \varepsilon_{n}} r_{1} J_{n}\left(k r_{1}\right)\left[Y_{n}^{\prime}(k \rho)-\mathrm{i} J_{n}^{\prime}(k \rho)\right]\left(a_{n} \cos n \phi+b_{n} \sin n \phi\right), & n=0,1,2, \ldots
\end{array}
$$

These turn out to be $u_{n}(\rho, \phi)=t_{n}(\rho, \phi)=0$ on account of $(2.25)$, so that the spurious modes (3.7) and (3.8) corresponding to the spurious eigenvalues hiding in the singular BIE are trivial null fields. However, from (2.27), the spurious modes (3.7) and (3.8) corresponding to the spurious eigenvalues hiding in the hypersingular BIE are not trivial. Even though the spurious mode is trivial theoretically, the contour distribution can be plotted numerically due to no true zero in numerical computation.

\section{Null-field formulations of BIEs}

In the above sections, the mechanisms of the spurious eigensolutions in the multiply connected domain were explored analytically using the degenerate kernels and the Fourier series expansions in the singular and hypersingular BIEs. To avoid singularity, a null-field approach may be employed. Firstly, we collocate $(2.14)$ at a point $\left(\rho_{1}, \phi_{1}\right)$, where $\rho_{1}<r_{1}, 0 \leqslant \phi_{1}<2 \pi$, by substituting the field point $\left(\rho_{1}, \phi_{1}\right)$ into $(2.14)$, and obtain the null-field equation

$$
\begin{aligned}
\sum_{n=0}^{\infty} \frac{1}{\varepsilon_{n}} \pi^{2} r_{2} J_{n}\left(k \rho_{1}\right)\left[Y_{n}\left(k r_{2}\right)-\mathrm{i} J_{n}\left(k r_{2}\right)\right]\left(p_{n} \cos n \phi_{1}+q_{n} \sin n \phi_{1}\right) \\
\\
\quad-\sum_{n=0}^{\infty} \frac{1}{\varepsilon_{n}} \pi^{2} r_{1} J_{n}\left(k \rho_{1}\right)\left[Y_{n}\left(k r_{1}\right)-\mathrm{i} J_{n}\left(k r_{1}\right)\right]\left(a_{n} \cos n \phi_{1}+b_{n} \sin n \phi_{1}\right)=0 .
\end{aligned}
$$

From (4.1), we obtain the relations between $a_{n}, b_{n}$ and $p_{n}, q_{n}$ as follows:

$$
\begin{aligned}
& p_{n}=\left(\frac{r_{1} J_{n}\left(k \rho_{1}\right)\left[Y_{n}\left(k r_{1}\right)-\mathrm{i} J_{n}\left(k r_{1}\right)\right]}{r_{2} J_{n}\left(k \rho_{1}\right)\left[Y_{n}\left(k r_{2}\right)-\mathrm{i} J_{n}\left(k r_{2}\right)\right]}\right) a_{n}, \quad n=0,1,2, \ldots, \\
& q_{n}=\left(\frac{r_{1} J_{n}\left(k \rho_{1}\right)\left[Y_{n}\left(k r_{1}\right)-\mathrm{i} J_{n}\left(k r_{1}\right)\right]}{r_{2} J_{n}\left(k \rho_{1}\right)\left[Y_{n}\left(k r_{2}\right)-\mathrm{i} J_{n}\left(k r_{2}\right)\right]}\right) b_{n}, \quad n=1,2, \ldots
\end{aligned}
$$

Note that (4.2) and (4.3) are mathematically equivalent to (2.18) and (2.19) if the common terms $J_{n}\left(k \rho_{1}\right)$ in the denominators and numerators of the right-hand sides of (4.2) and (4.3) and the common terms $J_{n}\left(k r_{1}\right)$ in the denominators and numerators of the right-hand sides of (2.18) and (2.19) are all cancelled out. 
Secondly, we collocate $(2.14)$ at another point $\left(\rho_{2}, \phi_{2}\right)$, where $\rho_{2}>r_{2}$ and $0 \leqslant \phi_{2}<2 \pi$, to obtain the null-field equation

$$
\begin{aligned}
\sum_{n=0}^{\infty} \frac{1}{\varepsilon_{n}} \pi^{2} r_{2} J_{n}\left(k r_{2}\right)\left[Y_{n}\left(k \rho_{2}\right)-\mathrm{i} J_{n}\left(k \rho_{2}\right)\right]\left(p_{n} \cos n \phi_{1}+q_{n} \sin n \phi_{1}\right) \\
-\sum_{n=0}^{\infty} \frac{1}{\varepsilon_{n}} \pi^{2} r_{1} J_{n}\left(k r_{1}\right)\left[Y_{n}\left(k \rho_{2}\right)-\mathrm{i} J_{n}\left(k \rho_{2}\right)\right]\left(a_{n} \cos n \phi_{1}+b_{n} \sin n \phi_{1}\right)=0
\end{aligned}
$$

from which follow the relations

$$
\begin{aligned}
& p_{n}=\left(\frac{r_{1} J_{n}\left(k r_{1}\right)\left[Y_{n}\left(k \rho_{2}\right)-\mathrm{i} J_{n}\left(k \rho_{2}\right)\right]}{r_{2} J_{n}\left(k r_{2}\right)\left[Y_{n}\left(k \rho_{2}\right)-\mathrm{i} J_{n}\left(k \rho_{2}\right)\right]}\right) a_{n}, \quad n=0,1,2, \ldots, \\
& q_{n}=\left(\frac{r_{1} J_{n}\left(k r_{1}\right)\left[Y_{n}\left(k \rho_{2}\right)-\mathrm{i} J_{n}\left(k \rho_{2}\right)\right]}{r_{2} J_{n}\left(k r_{2}\right)\left[Y_{n}\left(k \rho_{2}\right)-\mathrm{i} J_{n}\left(k \rho_{2}\right)\right]}\right) b_{n}, \quad n=1,2, \ldots
\end{aligned}
$$

Similarly, equations (4.5) and (4.6) are mathematically equivalent to (2.21) and (2.22) if the common terms $Y_{n}\left(k \rho_{2}\right)-\mathrm{i} J_{n}\left(k \rho_{2}\right)$ in the denominators and numerators of the right-hand sides of (4.5) and (4.6) and the common terms $Y_{n}\left(k r_{2}\right)-\mathrm{i} J_{n}\left(k r_{2}\right)$ in the denominators and numerators of the right-hand sides of (2.21) and (2.22) are all cancelled out. From (4.2), (4.3), (4.5) and (4.6), the formulation of null-field BIEs is shown to have the ability to solve the eigenproblem free from the singular and hypersingular integrals. Note that, from (4.2) and (4.3), the collocation with radius $\rho_{1}$ may fail in case of $J_{n}\left(k \rho_{1}\right)=0$. This indicates that the null-field formulation also results in spurious eigensolutions, as will be demonstrated in examples in $\S 8$.

The above is for the null-field formulation with the $U T$ kernels. For the aforementioned concept using the $L M$ kernels, the analysis is similar but the common terms are different.

\section{Fictitious boundary formulations of BIEs}

In order to avoid singularity, instead of the null-field formulations, the fictitious boundary formulations are another choice. In this section we present the fictitious BIEs based on the indirect method, first adopting the single-layer-potential approach and then the double-layer-potential approach.

For the single-layer-potential approach, the single-layer density $\phi$ is distributed on the inner and outer fictitious boundaries $B_{1}^{\prime}$ and $B_{2}^{\prime}$ and the field solutions are represented in terms of the single-layer potential,

$$
\begin{aligned}
& u(\boldsymbol{x})=\int_{B^{\prime}} U(\boldsymbol{s}, \boldsymbol{x}) \phi(\boldsymbol{s}) \mathrm{d} B(\boldsymbol{s}), \\
& t(\boldsymbol{x})=\int_{B^{\prime}} L(\boldsymbol{s}, \boldsymbol{x}) \phi(\boldsymbol{s}) \mathrm{d} B(\boldsymbol{s}),
\end{aligned}
$$

where the fictitious boundary $B^{\prime}$ involves the retracted boundary $B_{1}^{\prime}$ and the expanded boundary $B_{2}^{\prime}$. Expanding the boundary densities into the Fourier series, we 

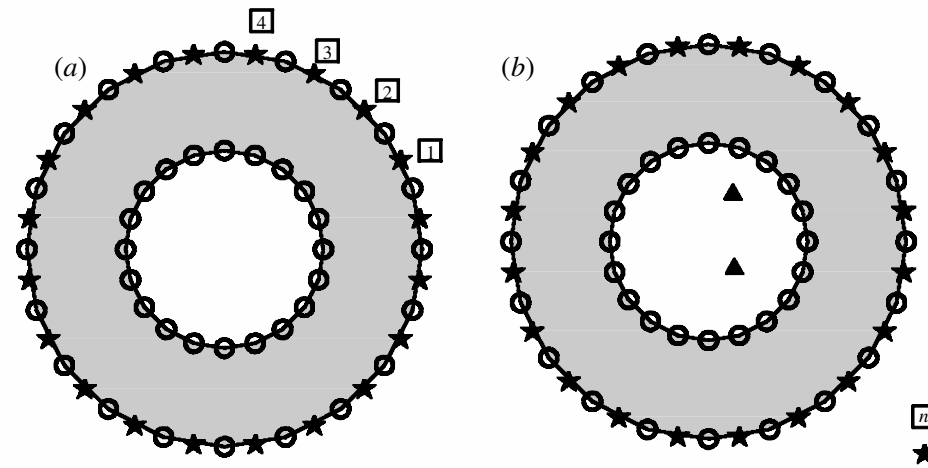

n element number

$\star$ collocation point

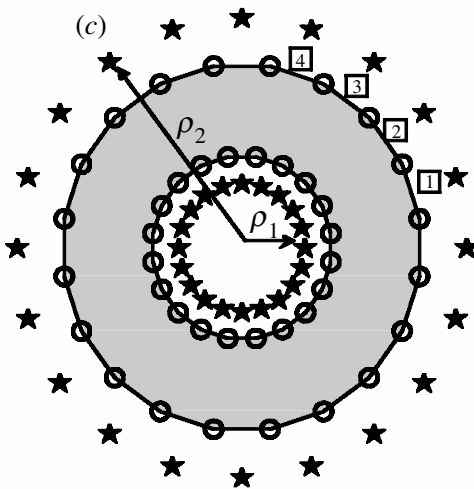

(1)

o node

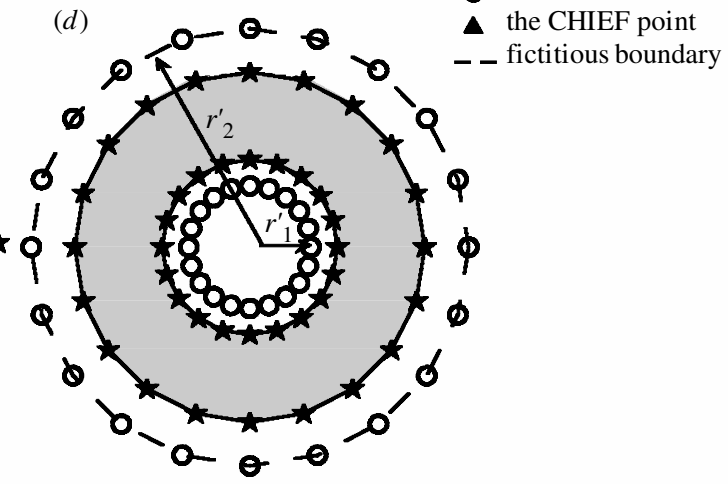

Figure 3. Four approaches: $(a)$ direct BEM; $(b)$ CHIEF method;

(c) null-field formulation; $(d)$ fictitious BEM.

have

$$
\begin{array}{ll}
\phi(s)=a_{0}^{\prime}+\sum_{n=1}^{N}\left(a_{n}^{\prime} \cos n \theta+b_{n}^{\prime} \sin n \theta\right), & s \in B_{1}^{\prime}, \\
\phi(s)=p_{0}^{\prime}+\sum_{n=1}^{N}\left(p_{n}^{\prime} \cos n \theta+q_{n}^{\prime} \sin n \theta\right), \quad s \in B_{2}^{\prime},
\end{array}
$$

where $a_{0}^{\prime}, p_{0}^{\prime} a_{n}^{\prime}, b_{n}^{\prime} p_{n}^{\prime}$ and $q_{n}^{\prime}(n=1,2, \ldots)$ are the Fourier coefficients. Considering the same Dirichlet problem as in the preceding sections, we have

$$
\begin{aligned}
0= & \int_{B^{\prime}} U(\boldsymbol{s}, \boldsymbol{x}) \phi(\boldsymbol{s}) \mathrm{d} B(\boldsymbol{s}) \\
= & \int_{B_{2}^{\prime}} U^{\mathrm{i}}(\boldsymbol{s}, \boldsymbol{x}) \phi(\boldsymbol{s}) \mathrm{d} B(\boldsymbol{s})+\int_{B_{1}^{\prime}} U^{\mathrm{e}}(\boldsymbol{s}, \boldsymbol{x}) \phi(\boldsymbol{s}) \mathrm{d} B(\boldsymbol{s}) \\
= & \sum_{n=0}^{N} \frac{1}{\varepsilon_{n}} \pi^{2} r_{2}^{\prime} J_{n}\left(k r_{1}\right)\left[Y_{n}\left(k r_{2}^{\prime}\right)-\mathrm{i} J_{n}\left(k r_{2}^{\prime}\right)\right]\left(p_{n}^{\prime} \cos n \phi+q_{n}^{\prime} \sin n \phi\right) \\
& \quad-\sum_{n=0}^{N} \frac{1}{\varepsilon_{n}} \pi^{2} r_{1}^{\prime} J_{n}\left(k r_{1}^{\prime}\right)\left[Y_{n}\left(k r_{1}\right)-\mathrm{i} J_{n}\left(k r_{1}\right)\right]\left(a_{n}^{\prime} \cos n \phi+p_{n}^{\prime} \sin n \phi\right), \quad \boldsymbol{x} \in B_{1},
\end{aligned}
$$


and

$$
\begin{aligned}
0= & \int_{B^{\prime}} U(\boldsymbol{s}, \boldsymbol{x}) \phi(\boldsymbol{s}) \mathrm{d} B(\boldsymbol{s}) \\
= & \int_{B_{2}^{\prime}} U^{\mathrm{i}}(\boldsymbol{s}, \boldsymbol{x}) \phi(\boldsymbol{s}) \mathrm{d} B(\boldsymbol{s})+\int_{B_{1}^{\prime}} U^{\mathrm{e}}(\boldsymbol{s}, \boldsymbol{x}) \phi(\boldsymbol{s}) \mathrm{d} B(\boldsymbol{s}) \\
= & \sum_{n=0}^{N} \frac{1}{\varepsilon_{n}} \pi^{2} r_{2}^{\prime} J_{n}\left(k r_{2}\right)\left[Y_{n}\left(k r_{2}^{\prime}\right)-\mathrm{i} J_{n}\left(k r_{2}^{\prime}\right)\right]\left(p_{n}^{\prime} \cos n \phi+q_{n}^{\prime} \sin n \phi\right) \\
& \quad-\sum_{n=0}^{N} \frac{1}{\varepsilon_{n}} \pi^{2} r_{1}^{\prime} J_{n}\left(k r_{1}^{\prime}\right)\left[Y_{n}\left(k r_{2}\right)-\mathrm{i} J_{n}\left(k r_{2}\right)\right]\left(a_{n}^{\prime} \cos n \phi+p_{n}^{\prime} \sin n \phi\right), \quad \boldsymbol{x} \in B_{2},
\end{aligned}
$$

where $r_{1}^{\prime}$ and $r_{2}^{\prime}$ are shown in figure $3 d$. From (5.5), we have the relations

$$
\begin{aligned}
& p_{n}^{\prime}=\left(\frac{r_{1}^{\prime} J_{n}\left(k r_{1}^{\prime}\right)\left[Y_{n}\left(k r_{1}\right)-\mathrm{i} J_{n}\left(k r_{1}\right)\right]}{r_{2}^{\prime} J_{n}\left(k r_{1}\right)\left[Y_{n}\left(k r_{2}^{\prime}\right)-\mathrm{i} J_{n}\left(k r_{2}^{\prime}\right)\right]}\right) a_{n}^{\prime}, \quad n=0,1,2 \ldots, \\
& q_{n}^{\prime}=\left(\frac{r_{1}^{\prime} J_{n}\left(k r_{1}^{\prime}\right)\left[Y_{n}\left(k r_{1}\right)-\mathrm{i} J_{n}\left(k r_{1}\right)\right]}{r_{2}^{\prime} J_{n}\left(k r_{1}\right)\left[Y_{n}\left(k r_{2}^{\prime}\right)-\mathrm{i} J_{n}\left(k r_{2}^{\prime}\right)\right]}\right) b_{n}^{\prime}, \quad n=1,2 \ldots
\end{aligned}
$$

Similarly, from (5.6), we have the relations

$$
\begin{aligned}
& p_{n}^{\prime}=\left(\frac{r_{1}^{\prime} J_{n}\left(k r_{1}^{\prime}\right)\left[Y_{n}\left(k r_{2}\right)-\mathrm{i} J_{n}\left(k r_{2}\right)\right]}{r_{2}^{\prime} J_{n}\left(k r_{2}\right)\left[Y_{n}\left(k r_{2}^{\prime}\right)-\mathrm{i} J_{n}\left(k r_{2}^{\prime}\right)\right]}\right) a_{n}^{\prime}, \quad n=0,1,2 \ldots, \\
& q_{n}^{\prime}=\left(\frac{r_{1}^{\prime} J_{n}\left(k r_{1}^{\prime}\right)\left[Y_{n}\left(k r_{2}\right)-\mathrm{i} J_{n}\left(k r_{2}\right)\right]}{r_{2}^{\prime} J_{n}\left(k r_{2}\right)\left[Y_{n}\left(k r_{2}^{\prime}\right)-\mathrm{i} J_{n}\left(k r_{2}^{\prime}\right)\right]}\right) b_{n}^{\prime}, \quad n=1,2 \ldots
\end{aligned}
$$

Combination of (5.7), (5.8), (5.9) and (5.10) yields the eigenequations

$$
J_{n}\left(k r_{1}^{\prime}\right)\left[J_{n}\left(k r_{1}\right) Y_{n}\left(k r_{2}\right)-J_{n}\left(k r_{2}\right) Y_{n}\left(k r_{1}\right)\right]=0, \quad n=0,1,2 \ldots
$$

It is seen from (5.11) that the spurious eigenvalues imbedded in the fictitious BIEs using the single-layer-potential approach are nothing but the values of $k$ that satisfy $J_{n}\left(k r_{1}^{\prime}\right)=0$. This indicates that the spurious eigenvalues depend on the location of the inner fictitious boundary $\left(r_{1}^{\prime}\right)$ chosen to solve the multiply connected problem. Although the spurious eigenvalues are different from those using the true inner boundary, the true eigenvalues are identical to those obtained by the other formulations.

For the double-layer-potential approach of the indirect method,

$$
\begin{aligned}
& u(\boldsymbol{x})=\int_{B^{\prime}} T(\boldsymbol{s}, \boldsymbol{x}) \psi(\boldsymbol{s}) \mathrm{d} B(\boldsymbol{s}), \\
& t(\boldsymbol{x})=\int_{B^{\prime}} M(\boldsymbol{s}, \boldsymbol{x}) \psi(\boldsymbol{s}) \mathrm{d} B(\boldsymbol{s}),
\end{aligned}
$$

where the double-layer density $\psi$ is distributed on the inner and outer fictitious boundaries $B_{1}^{\prime}$ and $B_{2}^{\prime}$. Expanding the boundary densities into the Fourier series, we 
have

$$
\begin{array}{ll}
\psi(s)=\bar{a}_{0}^{\prime}+\sum_{n=1}^{\infty}\left(\bar{a}_{n}^{\prime} \cos n \theta+\bar{b}_{n}^{\prime} \sin n \theta\right), \quad s \in B_{1}^{\prime}, \\
\psi(s)=\bar{p}_{0}^{\prime}+\sum_{n=1}^{\infty}\left(\bar{p}_{n}^{\prime} \cos n \theta+\bar{q}_{n}^{\prime} \sin n \theta\right), \quad s \in B_{2}^{\prime},
\end{array}
$$

where $\bar{a}_{0}^{\prime}, \bar{p}_{0}^{\prime}, \bar{a}_{n}^{\prime}, \bar{b}_{n}^{\prime}, \bar{p}_{n}^{\prime}$ and $\bar{q}_{n}^{\prime}(n=1,2, \ldots)$ are the Fourier coefficients. For the Dirichlet problem, collocating the equation at the points $\boldsymbol{x} \in B_{1}$ and $\boldsymbol{x} \in B_{2}$ yields

$$
\begin{array}{ll}
0=\int_{B_{2}^{\prime}} T^{\mathrm{i}}(\boldsymbol{s}, \boldsymbol{x}) \psi(\boldsymbol{s}) \mathrm{d} B(\boldsymbol{s})+\int_{B_{1}^{\prime}} T^{\mathrm{e}}(\boldsymbol{s}, \boldsymbol{x}) \psi(\boldsymbol{s}) \mathrm{d} B(\boldsymbol{s}), & \boldsymbol{x} \in B_{1}, \\
0=\int_{B_{2}^{\prime}} T^{\mathrm{i}}(\boldsymbol{s}, \boldsymbol{x}) \psi(\boldsymbol{s}) \mathrm{d} B(\boldsymbol{s})+\int_{B_{1}^{\prime}} T^{\mathrm{e}}(\boldsymbol{s}, \boldsymbol{x}) \psi(\boldsymbol{s}) \mathrm{d} B(\boldsymbol{s}), & \boldsymbol{x} \in B_{2},
\end{array}
$$

from which follow the relations

$$
\begin{aligned}
& \bar{p}_{n}^{\prime}=\left(\frac{r_{1}^{\prime} J_{n}^{\prime}\left(k r_{1}^{\prime}\right)\left[Y_{n}\left(k r_{1}\right)-\mathrm{i} J_{n}\left(k r_{1}\right)\right]}{r_{2}^{\prime} J_{n}\left(k r_{1}\right)\left[Y_{n}\left(k r_{2}^{\prime}\right)-\mathrm{i} J_{n}\left(k r_{2}^{\prime}\right)\right]}\right) \bar{a}_{n}^{\prime}, \quad n=0,1,2 \ldots, \\
& \bar{q}_{n}^{\prime}=\left(\frac{r_{1}^{\prime} J_{n}^{\prime}\left(k r_{1}^{\prime}\right)\left[Y_{n}\left(k r_{1}\right)-\mathrm{i} J_{n}\left(k r_{1}\right)\right]}{r_{2}^{\prime} J_{n}\left(k r_{1}\right)\left[Y_{n}\left(k r_{2}^{\prime}\right)-\mathrm{i} J_{n}\left(k r_{2}^{\prime}\right)\right]}\right) \bar{b}_{n}^{\prime}, \quad n=1,2 \ldots,
\end{aligned}
$$

and

$$
\begin{aligned}
& \bar{p}_{n}^{\prime}=\left(\frac{r_{1}^{\prime} J_{n}^{\prime}\left(k r_{1}^{\prime}\right)\left[Y_{n}\left(k r_{2}\right)-\mathrm{i} J_{n}\left(k r_{2}\right)\right]}{r_{2}^{\prime} J_{n}\left(k r_{2}\right)\left[Y_{n}\left(k r_{2}^{\prime}\right)-\mathrm{i} J_{n}\left(k r_{2}^{\prime}\right)\right]}\right) \bar{a}_{n}^{\prime}, \quad n=0,1,2 \ldots, \\
& \bar{q}_{n}^{\prime}=\left(\frac{r_{1}^{\prime} J_{n}^{\prime}\left(k r_{1}^{\prime}\right)\left[Y_{n}\left(k r_{2}\right)-\mathrm{i} J_{n}\left(k r_{2}\right)\right]}{r_{2}^{\prime} J_{n}\left(k r_{2}\right)\left[Y_{n}\left(k r_{2}^{\prime}\right)-\mathrm{i} J_{n}\left(k r_{2}^{\prime}\right)\right]}\right) \bar{b}_{n}^{\prime}, \quad n=1,2 \ldots,
\end{aligned}
$$

respectively. Combining (5.18), (5.19), (5.20) and (5.21), we have the eigenequations

$$
J_{n}^{\prime}\left(k r_{1}^{\prime}\right)\left[J_{n}\left(k r_{1}\right) Y_{n}\left(k r_{2}\right)-J_{n}\left(k r_{2}\right) Y_{n}\left(k r_{1}\right)\right]=0, \quad n=0,1,2 \ldots
$$

According to (5.22), the spurious eigenvalues imbedded in the fictitious BIE using the double-layer-potential approach are nothing but the values of $k$ that satisfy $J_{n}^{\prime}\left(k r_{1}^{\prime}\right)=0$. Comparing (5.11) with (5.22), we find that the spurious eigenvalues depend on the location of the inner fictitious boundary $\left(r_{1}^{\prime}\right)$ for the multiply connected problem, no matter what one of the two approaches is used. Moreover, true eigensolutions are always conserved and spurious eigensolutions are also imbedded in the fictitious BIEs regardless of whether the singular-layer or double-layer approach is used.

\section{CHIEF treatment for spurious eigensolutions}

According to (2.18), (2.19), (2.21) and (2.22), the $k$ values that satisfy $J_{n}\left(k r_{1}\right)=0$ are the spurious eigenvalues. Therefore, the constraint becomes a trivial equation. Chen et al. $(2001 c)$ have used the modified Burton-Miller methods $(\mathrm{i} k U(\boldsymbol{s}, \boldsymbol{x})+L(\boldsymbol{s}, \boldsymbol{x}))$ or its real part $\left(\mathrm{i} k\left[U_{r}(\boldsymbol{s}, \boldsymbol{x})\right]+\left[L_{r}(\boldsymbol{s}, \boldsymbol{x})\right]\right)$ to sort out the true eigenvalues. In this section 


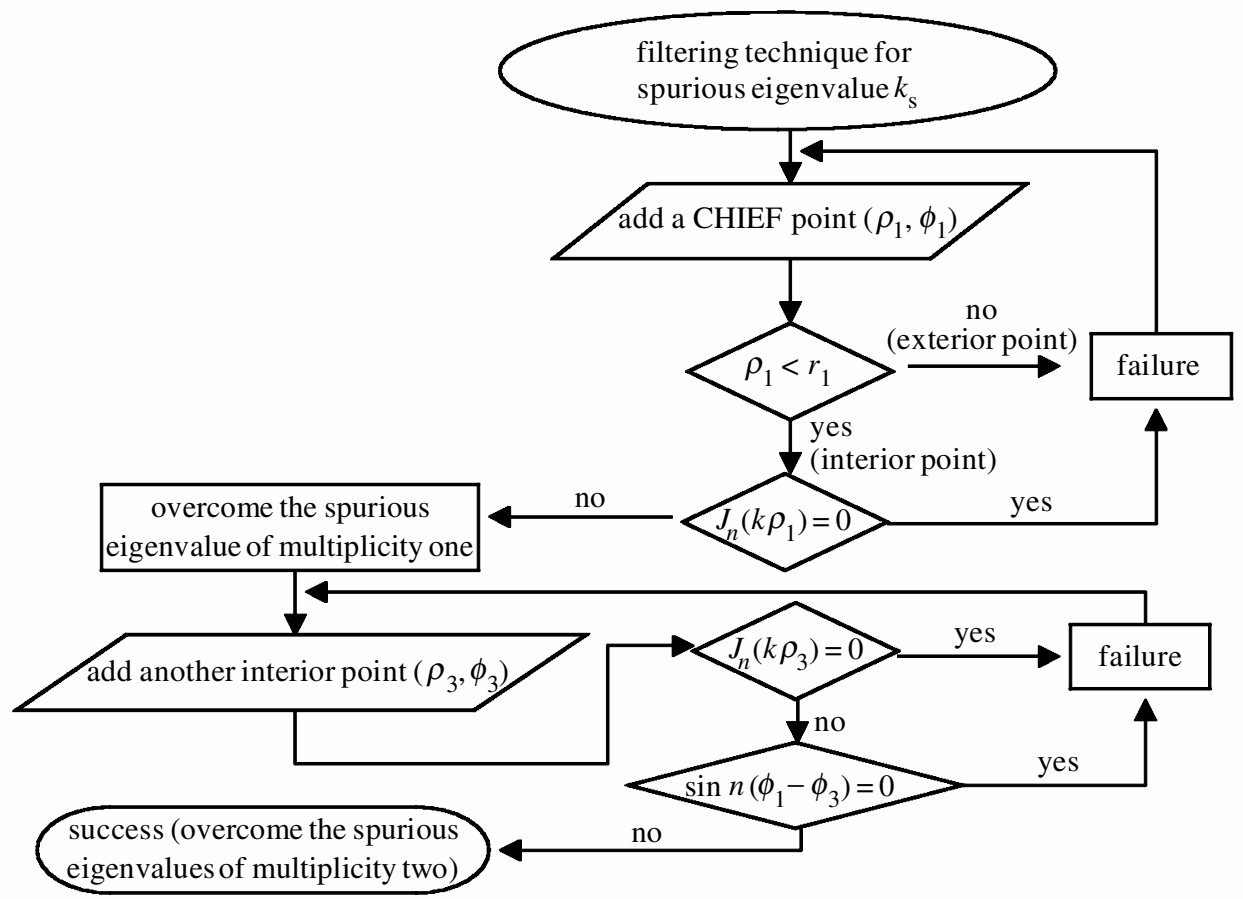

Figure 4. Flowchart of the CHIEF method.

we employ the CHIEF method to deal with the problem of spurious eigenvalues. In choosing the additional points, two options may be considered: one is to put the points in the region inside the inner circle (the CHIEF points) (Chen et al. 2001b); and the other is to put them in the region outside the outer circle (the CHEEF points) (Chen et al. 2001a).

Firstly, we choose a CHIEF point $\left(\rho_{1}, \phi_{1}\right)$, obtaining specific equations (4.2) and (4.3) for the point. Combining (2.21), (2.22), (4.2) and (4.3), we have

$$
J_{n}\left(k \rho_{1}\right)\left[J_{n}\left(k r_{2}\right) Y_{n}\left(k r_{1}\right)-J_{n}\left(k r_{1}\right) Y_{n}\left(k r_{1}\right)\right]=0, \quad n=0,1,2, \ldots
$$

Comparing (6.1) with the true eigenequation (2.23), we find that the CHIEF method works by adding a point in the area inside the inner circle to overcome the spurious-eigenvalue problem if the selected point is not located on the position where $J_{n}\left(k \rho_{1}\right)=0$. It is interesting that the failure criterion is the same as that of the null-field formulation discussed in $\oint 4$. Consequently, when the $k_{\mathrm{s}}$ values satisfy the spurious eigenequations, $J_{n}\left(k_{\mathrm{s}} r_{1}\right)=0$, we can resort to (6.1) to obtain a valid constraint where $J_{n}\left(k_{\mathrm{s}} \rho_{1}\right) \neq 0$.

Secondly, we choose a CHEEF point $\left(\rho_{2}, \phi_{2}\right)$ outside the outer circle, obtaining specific equations (4.5) and (4.6) for the point. Combining (2.21), (2.22), (4.5) and (4.6), we have

$$
J_{n}\left(k r_{1}\right) J_{n}\left(k r_{2}\right)=J_{n}\left(k r_{1}\right) J_{n}\left(k r_{2}\right), \quad n=0,1,2, \ldots
$$

Equation (6.2) provides merely trivial information for any value of $k$, since it is an identity. Hence it fails by adding a point in the area outside the outer circle.

When the spurious eigenvalue is a root of multiplicity two, we need two independent constraints to filter out the spurious eigenvalues. Because one added point 
supplies at most one constraint, an additional point is required. In the preceding discussion, the location of the added point must be in the region inside the inner circle in order to obtain independent constraints. Therefore, we add another point $\left(\rho_{3}, \phi_{3}\right)$ in this region to filter out the spurious eigenvalue of multiplicity two. Substituting the field point $\left(\rho_{3}, \phi_{3}\right)$ into $(2.14)$, we have

$$
\begin{aligned}
\sum_{n=0}^{\infty} \frac{1}{\varepsilon_{n}} \pi^{2} r_{2} J_{n}\left(k \rho_{3}\right)\left[Y_{n}\left(k r_{2}\right)-\mathrm{i} J_{n}\left(k r_{2}\right)\right]\left(p_{n} \cos n \phi_{3}+q_{n} \sin n \phi_{3}\right) \\
\quad-\sum_{n=0}^{\infty} \frac{1}{\varepsilon_{n}} \pi^{2} r_{1} J_{n}\left(k \rho_{3}\right)\left[Y_{n}\left(k r_{1}\right)-\mathrm{i} J_{n}\left(k r_{1}\right)\right]\left(a_{n} \cos n \phi_{3}+b_{n} \sin n \phi_{3}\right)=0 .
\end{aligned}
$$

Multiplication of the coefficients $\mu$ and $\nu$ with (4.1) and (6.3), respectively, and combination yield

$$
\begin{aligned}
& \sum_{n=0}^{\infty} \frac{1}{\varepsilon_{n}} \pi^{2} r_{2}\left[Y_{n}\left(k r_{2}\right)-\mathrm{i} J_{n}\left(k r_{2}\right)\right]\left[\mu J_{n}\left(k \rho_{1}\right) \cos n \phi_{1}+\nu J_{n}\left(k \rho_{3}\right) \cos n \phi_{3}\right] p_{n} \\
& +\sum_{n=1}^{\infty} \frac{1}{2} \pi^{2} r_{2}\left[Y_{n}\left(k r_{2}\right)-\mathrm{i} J_{n}\left(k r_{2}\right)\right]\left[\mu J_{n}\left(k \rho_{1}\right) \sin n \phi_{1}+\nu J_{n}\left(k \rho_{3}\right) \sin n \phi_{3}\right] q_{n} \\
& \quad-\sum_{n=0}^{\infty} \frac{1}{\varepsilon_{n}} \pi^{2} r_{1}\left[Y_{n}\left(k r_{1}\right)-\mathrm{i} J_{n}\left(k r_{1}\right)\right]\left[\mu J_{n}\left(k \rho_{1}\right) \cos n \phi_{1}+\nu J_{n}\left(k \rho_{3}\right) \cos n \phi_{3}\right] a_{n} \\
& \quad-\sum_{n=1}^{\infty} \frac{1}{2} \pi^{2} r_{1}\left[Y_{n}\left(k r_{1}\right)-\mathrm{i} J_{n}\left(k r_{1}\right)\right]\left[\mu J_{n}\left(k \rho_{1}\right) \sin n \phi_{1}+\nu J_{n}\left(k \rho_{3}\right) \sin n \phi_{3}\right] b_{n}=0 .
\end{aligned}
$$

On the basis of linear algebra, the two equations for the CHIEF points are independent if and only if the coefficients of (6.4), $\mu$ and $\nu$, vanish for any situation. The terms of $\left[Y_{n}\left(k r_{1}\right)-\mathrm{i} J_{n}\left(k r_{1}\right)\right]$ and $\left[Y_{n}\left(k r_{2}\right)-\mathrm{i} J_{n}\left(k r_{2}\right)\right]$ are never zeros for any $k$; hence we have

$$
\left[\begin{array}{ll}
J_{n}\left(k \rho_{1}\right) \sin n \phi_{1} & J_{n}\left(k \rho_{3}\right) \sin n \phi_{3} \\
J_{n}\left(k \rho_{1}\right) \cos n \phi_{1} & J_{n}\left(k \rho_{3}\right) \cos n \phi_{3}
\end{array}\right]\left\{\begin{array}{c}
\mu \\
\nu
\end{array}\right\}=\left\{\begin{array}{l}
0 \\
0
\end{array}\right\} .
$$

If the determinant of the matrix in (6.5) vanishes, the coefficients $\mu$ and $\nu$ can be arbitrary, i.e. equations (4.1) and (6.3) do not provide two independent constraints. In this case, the intersection angle $\phi_{1}-\phi_{3}$ between the two selected points satisfying

$$
\sin n\left(\phi_{1}-\phi_{3}\right)=0 \quad \text { or } \quad \phi_{1}-\phi_{3}=\frac{\pi}{n}, \quad n=1,2,3, \ldots,
$$

makes the two equations dependent. Besides, the CHIEF point $\left(\rho_{3}, \phi_{3}\right)$ does not nullify $J_{n}\left(k \rho_{3}\right)$. Therefore, we must avoid this point in order to filter out the spurious eigenvalues of multiplicity two effectively. The flowchart of the CHIEF method is shown in figure 4. Although the above CHIEF treatment and discussions were developed for the dual BIEs formulation, almost the same treatments and discussions can be applied to deal with the spurious eigenvalues occurring in the null-field and fictitious BIEs formulations. 


\section{Detection of spurious and true eigenvalues in BEM formulations using SVD-updating techniques and Fredholm's alternative theorem}

In this section, the true and spurious eigensolutions will be detected and distinguished. Although we deal with the eigenproblem subject to homogeneous boundary, the following derivation may resort to the case of the non-homogeneous boundary condition by using the concept of spurious resonance. Two principles are adopted here. The spurious eigensolution depends on the formulation instead of the types of boundary conditions. On the contrary, the true solutions (eigenvalue and eigenmode) are embedded in any formulation and are different for the Dirichlet and Neumann problems.

(a) Spurious eigenvalues in direct BEMs detected by SVD-updating documents

In the BEM implementation, the singular BIE (2.2) is discretized to

$$
[U]\{t\}=[T]\{u\} \text {. }
$$

Let us consider two problems with non-homogeneous boundary conditions as follows,

$$
\begin{aligned}
{[U]\{t\} } & =[T]\{\bar{u}\}=\left\{\bar{b}_{\mathrm{D}}\right\} \quad \text { for the Dirichlet problem, } \\
{[U]\{\bar{t}\} } & =[T]\{u\}=\left\{\bar{b}_{\mathrm{N}}\right\} \quad \text { for the Neumann problem, }
\end{aligned}
$$

where $\{\bar{u}\}$ and $\{\bar{t}\}$ are specified boundary data, and the subscripts (or superscripts) ' $\mathrm{D}$ ' and ' $\mathrm{N}$ ' indicate the Dirichlet and Neumann problems, respectively. Since spurious solution depends on the formulation instead of the types of boundary condition, spurious eigenvalues are found embedded in both the Dirichlet problem (7.2) and the Neumann problem (7.3) in the case of spurious resonance, both of which have used the same UT equation (7.1). According to the Fredholm alternative theorem, the existence of the solution (corresponding to each spurious eigenvalue $k_{\mathrm{s}}$ ) to $(7.2)$ (respectively, (7.3)) implies

$$
\begin{array}{ll}
\left\{\bar{b}_{\mathrm{D}}\right\}^{\mathrm{H}}\left\{\phi_{\mathrm{s}}^{\mathrm{D}}\right\}=0 & \text { for the Dirichlet problem, } \\
\left\{\bar{b}_{\mathrm{N}}\right\}^{\mathrm{H}}\left\{\phi_{\mathrm{s}}^{\mathrm{N}}\right\}=0 & \text { for the Neumann problem, }
\end{array}
$$

where the superscript ' $H$ ' denotes the Hermitian conjugate and $\left\{\phi_{\mathrm{s}}^{\mathrm{D}}\right\}$ and $\left\{\phi_{\mathrm{s}}^{\mathrm{N}}\right\}$ are the spurious modes that satisfy the adjoint systems

$$
\begin{aligned}
& {\left[U\left(k_{\mathrm{s}}\right)\right]^{\mathrm{H}}\left\{\phi_{\mathrm{s}}^{\mathrm{D}}\right\}=\{0\} \quad \text { for the Dirichlet problem, }} \\
& {\left[T\left(k_{\mathrm{s}}\right)\right]^{\mathrm{H}}\left\{\phi_{\mathrm{s}}^{\mathrm{N}}\right\}=\{0\} \quad \text { for the Neumann problem. }}
\end{aligned}
$$

Substituting (7.2) and (7.3) into (7.4) and (7.5), respectively, we obtain

$$
\begin{aligned}
\{\bar{u}\}^{\mathrm{H}}\left[T\left(k_{\mathrm{s}}\right)\right]^{\mathrm{H}}\left\{\phi_{\mathrm{s}}^{\mathrm{D}}\right\}=0 & \text { for the Dirichlet problem, } \\
\{\bar{t}\}^{\mathrm{H}}\left[U\left(k_{\mathrm{s}}\right)\right]^{\mathrm{H}}\left\{\phi_{\mathrm{s}}^{\mathrm{N}}\right\}=0 & \text { for the Neumann problem. }
\end{aligned}
$$

Since $\{\bar{u}\}$ and $\{\bar{t}\}$ are arbitrary, equations (7.8) and (7.9) imply

$$
\begin{aligned}
& {\left[T\left(k_{\mathrm{s}}\right)\right]^{\mathrm{H}}\left\{\phi_{\mathrm{s}}^{\mathrm{D}}\right\}=\{0\} \quad \text { for the Dirichlet problem, }} \\
& {\left[U\left(k_{\mathrm{s}}\right)\right]^{\mathrm{H}}\left\{\phi_{\mathrm{s}}^{\mathrm{N}}\right\}=\{0\} \quad \text { for the Neumann problem. }}
\end{aligned}
$$


Combining (7.10) and (7.11) with (7.6) and (7.7), respectively, we have

$$
\begin{aligned}
& {\left[\begin{array}{l}
U^{\mathrm{H}}\left(k_{\mathrm{s}}\right) \\
T^{\mathrm{H}}\left(k_{\mathrm{s}}\right)
\end{array}\right]\left\{\phi_{\mathrm{s}}^{\mathrm{D}}\right\}=\{0\} \quad \text { for the Dirichlet problem, }} \\
& {\left[\begin{array}{l}
U^{\mathrm{H}}\left(k_{\mathrm{s}}\right) \\
T^{\mathrm{H}}\left(k_{\mathrm{s}}\right)
\end{array}\right]\left\{\phi_{\mathrm{s}}^{\mathrm{N}}\right\}=\{0\} \quad \text { for the Neumann problem. }}
\end{aligned}
$$

Comparing (7.12) with (7.13), we find that the spurious modes for the Dirichlet and Neumann problems are identical, $\left(\phi_{\mathrm{s}}^{\mathrm{D}}=\phi_{\mathrm{s}}^{\mathrm{N}}=\phi_{\mathrm{s}}\right)$, and that

$$
\left[\begin{array}{l}
U^{\mathrm{H}}\left(k_{\mathrm{s}}\right) \\
T^{\mathrm{H}}\left(k_{\mathrm{s}}\right)
\end{array}\right]\left\{\phi_{\mathrm{s}}\right\}=\{0\} .
$$

Taking the Hermitian conjugation with respect to (7.14), we have

$$
\left\{\phi_{\mathrm{s}}\right\}^{\mathrm{H}}\left[U\left(k_{\mathrm{s}}\right) \quad T\left(k_{\mathrm{s}}\right)\right]=\{0\}^{\mathrm{H}} .
$$

The $[T]$ matrix can be thought to be the updating document with the $[U]$ matrix in $(7.15)$, while the $[T]^{\mathrm{H}}$ matrix is the updating term with the $[U]^{\mathrm{H}}$ matrix in $(7.14)$. From the preceding argument, the two matrices $[U]$ and $[T]$ have the same spurious mode $\left\{\phi_{\mathrm{s}}\right\}$ corresponding to each spurious eigenvalue $k_{\mathrm{s}}$.

Similarly, the $L M$ method has the same spurious mode $\left(\bar{\phi}_{\mathrm{s}}^{\mathrm{D}}=\bar{\phi}_{\mathrm{s}}^{\mathrm{N}}=\bar{\phi}_{\mathrm{s}}\right)$ corresponding to each spurious eigenvalue $\bar{k}_{\mathrm{s}}$ for the Dirichlet and Neumann problems as shown below,

$$
\left[\begin{array}{c}
L^{\mathrm{H}}\left(\bar{k}_{\mathrm{s}}\right) \\
M^{\mathrm{H}}\left(\bar{k}_{\mathrm{s}}\right)
\end{array}\right]\left\{\bar{\phi}_{\mathrm{s}}\right\}=\{0\}, \quad\left\{\bar{\phi}_{\mathrm{s}}\right\}^{\mathrm{H}}\left[L\left(\bar{k}_{\mathrm{s}}\right) \quad M\left(\bar{k}_{\mathrm{s}}\right)\right]=\{0\}^{\mathrm{H}} .
$$

Note that $\left\{\bar{\phi}_{\mathrm{s}}\right\}$ is the spurious mode resulting from the $L M$ method, whereas $\left\{\phi_{\mathrm{s}}\right\}$ is the spurious mode resulting from the $U T$ method. By the same token as in the $U T$ method, the $[L]$ and $[M]$ matrices have the same spurious eigenvalues. In other words, the spurious eigenvalues are related to those formulated by the UT method or by the $L M$ method rather than related to the boundary condition of the Dirichlet type or the Neumann type.

To detect the spurious eigenvalues, we merge the $[U]$ and $[T]$ matrices to form the so-called updating document,

$$
[B(k)]=[U(k) \quad T(k)] .
$$

Then applying the SVD leads to

$$
[B(k)]=\left[\Phi_{B}\right]\left[\Sigma_{B}\right]\left[\Psi_{B}\right]^{\mathrm{H}},
$$

where $\left[\Sigma_{B}\right]$ is a diagonal matrix with positive or zero singular values as diagonal elements and $\left[\Phi_{B}\right]$ and $\left[\Psi_{B}\right]$ are the left and right unitary matrices, respectively. Thus the minimum singular value of $[B(k)]$ as a (numerical) function of $k$ can be used to find the spurious eigenvalues $k_{\mathrm{s}}$ and then the spurious modes $\left\{\phi_{\mathrm{s}}\right\}$. 
(b) True eigenvalues in direct BEMs detected by SVD-updating terms

In this subsection we use the SVD-updating-term technique to detect true eigenvalues $k_{\mathrm{t}}$ obtained from the direct BEMs. First let us consider that the true solution must be embedded in

$$
\left.\begin{array}{l}
{\left[U\left(k_{\mathrm{t}}\right)\right]\{t\}=\{0\},} \\
{\left[L\left(k_{\mathrm{t}}\right)\right]\{t\}=\{0\}}
\end{array}\right\} \quad \text { for the homogeneous Dirichlet problem, }
$$

which indicates that both the $[U]$ and $[L]$ matrices have a zero singular value corresponding to the right unitary vector $\{t\}$. This finding guides us to merge the two equations together,

$$
\left[D\left(k_{\mathrm{t}}\right)\right]\{t\}=\{0\}
$$

where

$$
\left[D\left(k_{\mathrm{t}}\right)\right]=\left[\begin{array}{l}
U\left(k_{\mathrm{t}}\right) \\
L\left(k_{\mathrm{t}}\right)
\end{array}\right]
$$

and apply the SVD,

$$
[D(k)]=\left[\Phi_{\mathrm{D}}\right]\left[\Sigma_{\mathrm{D}}\right]\left[\Psi_{\mathrm{D}}\right]^{\mathrm{H}} .
$$

Theoretically, the column vector of $\left[\Psi^{\mathrm{D}}\right]$ corresponding to the zero singular value in $\left[\Sigma^{\mathrm{D}}\right]$ is nothing but the true boundary eigenmode $\{t\}$. By plotting the minimum singular value of $[D(k)]$ versus $k$, one has a curve that drops at the positions of true eigenvalues.

The SVD-updating-term technique can also be applied to the Neumann problem; equations (7.20) and (7.21) are thus replaced by

$$
[N(k)]\{u\}=\{0\}
$$

where

$$
[N(k)]=\left[\begin{array}{c}
T(k) \\
M(k)
\end{array}\right] .
$$

To detect true eigenvalues, a similar procedure for the minimum singular value of matrix $[N(k)]$ versus $k$ must be developed.

(c) True eigenvalues in indirect BEMs detected by SVD-updating documents

For the indirect fictitious BEMs, equations (5.1), (5.2), (5.12) and (5.13) are discretized to

$$
\begin{array}{ll}
\{u\}=[U]\{\phi\}, & \{t\}=[L]\{\phi\} \quad \text { using the single-layer-potential approach, } \\
\{u\}=[T]\{\psi\}, & \{t\}=[M]\{\psi\} \quad \text { using the double-layer-potential approach, }
\end{array}
$$

after discretization. According to the Fredholm alternative theorem, the existence of solution $\{\phi\}$ in (7.25) for the non-homogeneous Dirichlet problem $\left(u=\bar{u}\right.$ on $\left.B^{\prime}\right)$ implies

$$
\{\bar{u}\}^{\mathrm{H}}\left\{\eta_{\mathrm{s}}\right\}=0,
$$

where $\eta_{\mathrm{s}}$ satisfies

$$
\left[U\left(k_{\mathrm{t}}\right)\right]^{\mathrm{H}}\left\{\eta_{\mathrm{s}}\right\}=\{0\},
$$


and the subscript 's' of $\eta$ indicates here the single-layer-potential approach. Substituting (7.26) into (7.27), we obtain

$$
\{\psi\}^{\mathrm{H}}\left[T\left(k_{\mathrm{t}}\right)\right]^{\mathrm{H}}\left\{\eta_{\mathrm{s}}\right\}=0 .
$$

Since $\{\psi\}$ can be arbitrary for the arbitrary $\{\bar{u}\}$, equation (7.29) implies

$$
\left[T\left(k_{\mathrm{t}}\right)\right]^{\mathrm{H}}\left\{\eta_{\mathrm{s}}\right\}=\{0\} .
$$

Combining (7.28) with (7.30), we have

$$
\left[\begin{array}{l}
U^{\mathrm{H}}\left(k_{\mathrm{t}}\right) \\
T^{\mathrm{H}}\left(k_{\mathrm{t}}\right)
\end{array}\right]\left\{\eta_{\mathrm{s}}\right\}=\{0\} .
$$

Similarly, the existence of solution $\{\psi\}$ to (7.26) implies

$$
\{\bar{u}\}^{\mathrm{H}}\left\{\eta_{\mathrm{d}}\right\}=0 \text {, }
$$

where $\eta_{\mathrm{d}}$ satisfies

$$
\left[T\left(k_{\mathrm{t}}\right)\right]^{\mathrm{H}}\left\{\eta_{\mathrm{d}}\right\}=\{0\},
$$

and the subscript ' $\mathrm{d}$ ' of $\eta$ indicates the double-layer-potential approach. Substituting (7.25) into (7.32), we obtain

$$
\{\phi\}^{\mathrm{H}}\left[U\left(k_{\mathrm{t}}\right)\right]^{\mathrm{H}}\left\{\eta_{\mathrm{d}}\right\}=0 .
$$

Since $\{\phi\}$ can be arbitrary for the arbitrary $\{\bar{u}\}$, equation (7.34) implies

$$
\left[U\left(k_{\mathrm{t}}\right)\right]^{\mathrm{H}}\left\{\eta_{\mathrm{d}}\right\}=\{0\} .
$$

Combining (7.33) with (7.35), we have

$$
\left[\begin{array}{l}
U^{\mathrm{H}}\left(k_{\mathrm{t}}\right) \\
T^{\mathrm{H}}\left(k_{\mathrm{t}}\right)
\end{array}\right]\left\{\eta_{\mathrm{d}}\right\}=\{0\} .
$$

Comparing (7.31) with (7.36), we find

$$
\left\{\eta_{\mathrm{s}}\right\}=\left\{\eta_{\mathrm{d}}\right\}=\{\eta\}
$$

Hence, combining (7.28) and (7.33) gives

$$
\left[\begin{array}{l}
U^{\mathrm{H}}\left(k_{\mathrm{t}}\right) \\
T^{\mathrm{H}}\left(k_{\mathrm{t}}\right)
\end{array}\right]\{\eta\}=\{0\} .
$$

To detect the true eigenvalues, we plot the minimum singular value of the assembled matrix

$$
\left[\begin{array}{l}
U^{\mathrm{H}}(k) \\
T^{\mathrm{H}}(k)
\end{array}\right]
$$

versus $k$, and the curve drops show the positions of the true eigenvalues.

The technique of the SVD-updating document can be extended to the Neumann problem; the minimum singular value for the assembled matrix

$$
\left[\begin{array}{c}
L^{\mathrm{H}}(k) \\
M^{\mathrm{H}}(k)
\end{array}\right]
$$

versus $k$ is plotted, and the drops in the curve are also found at the positions of true eigenvalues. 
(d) Spurious eigenvalues in indirect BEMs detected by SVD-updating terms

In this subsection we examine the spurious eigensolutions of the indirect BEMs. First let us consider the homogeneous Dirichlet and Neumann problem,

$$
\begin{aligned}
& {\left[U\left(k_{\mathrm{s}}\right)\right]\{\phi\}=\{0\},} \\
& {\left[L\left(k_{\mathrm{s}}\right)\right]\{\phi\}=\{0\},}
\end{aligned}
$$

to detect the spurious eigenvalues occurring in the single-layer-potential approach. Combining (7.39) and (7.40), we have

$$
\left[\begin{array}{c}
U\left(k_{\mathrm{s}}\right) \\
L\left(k_{\mathrm{s}}\right)
\end{array}\right]\{\phi\}=\{0\} .
$$

The minimum singular value of the assembled matrix

$$
\left[\begin{array}{l}
U(k) \\
L(k)
\end{array}\right]
$$

versus $k$ is then plotted, the local minima of the resulted curve revealing the spurious eigenvalues $k_{\mathrm{s}}$. Next let us consider

$$
\left[\begin{array}{c}
T\left(k_{\mathrm{s}}\right) \\
M\left(k_{\mathrm{s}}\right)
\end{array}\right]\{\psi\}=\{0\}
$$

to detect the spurious eigenvalues occurring in the double-layer-potential approach. The minimum singular value of the assembled matrix

$$
\left[\begin{array}{l}
T(k) \\
M(k)
\end{array}\right]
$$

versus $k$ can be similarly examined to find the spurious eigenvalues $k_{\mathrm{s}}$.

\section{Numerical examples}

In order to check the validity of the CHIEF method, the null-field formulation and the fictitious BEM, we consider three numerical examples: one annular cavity, one eccentric cavity and one hexagonal-cavity problem.

\section{(a) Annular cavity}

(i) Singular and hypersingular BEMs

The radii of the inner and outer boundaries of the annular cavity are $0.5 \mathrm{~m}$ and $2 \mathrm{~m}$, respectively. Figure 5 shows the minimum singular value, $\sigma_{1}$, versus $k$ for the $[U]$, $[L],[T]$ and $[M]$ matrices. As predicted theoretically, the true eigenvalues of the Dirichlet problem are commonly imbedded in the $[U]$ and $[L]$ matrices, although the computational results for lower frequencies were reported to be not good (Hsiao \& Wendland 2000), while we easily find the true eigenvalues of the Neumann problem in the $[T]$ and $[M]$ matrices. Since spurious eigenvalues vary with different formulations instead of types of boundary conditions, the location of the common dips in the $[U]$ and $[T]$ matrices shows the spurious eigenvalues for the singular (UT) formulation. 

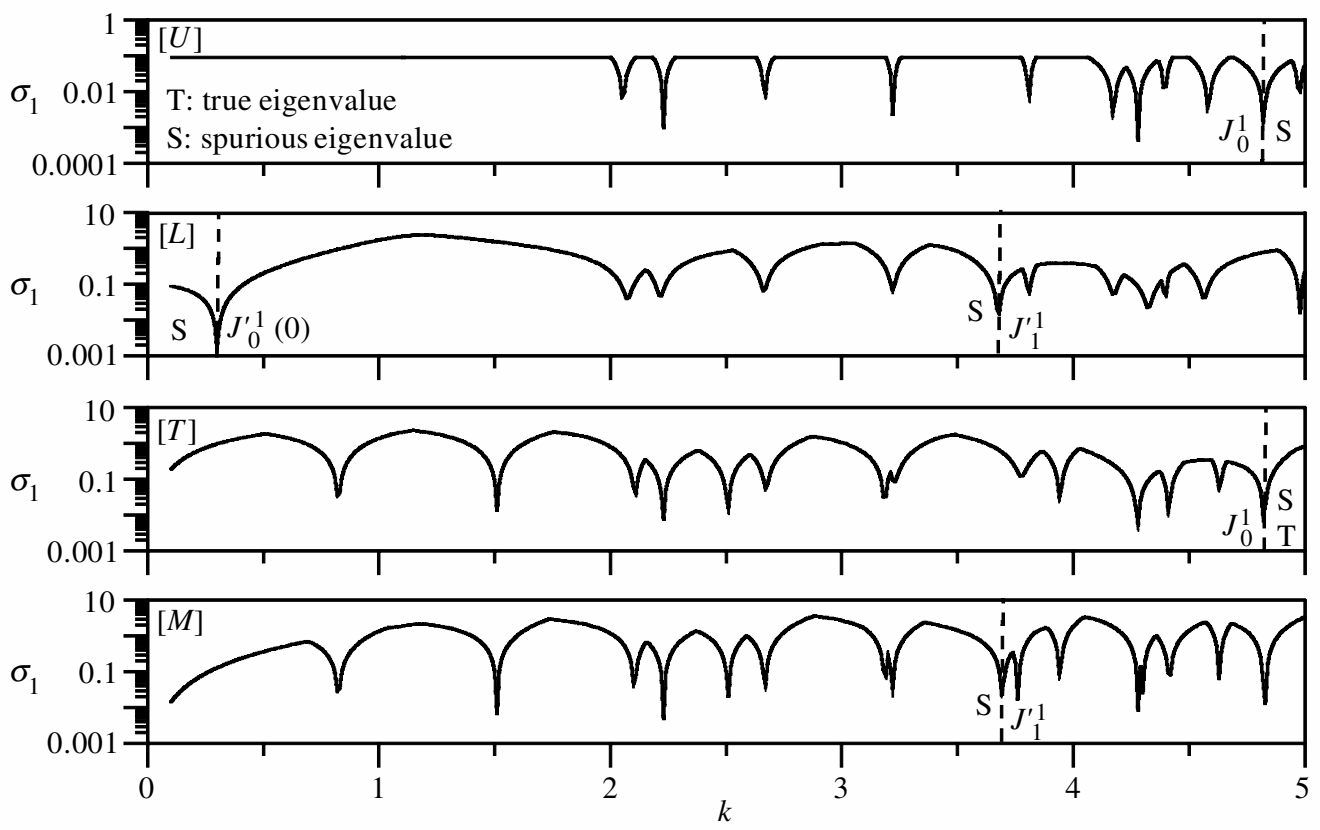

Figure 5. The minimum singular values $\left(\sigma_{1}\right)$ versus $k$ for the $[U],[L],[T]$ and $[M]$ matrices.

In the same way, the hypersingular $(L M)$ formulation results in another set of spurious eigenvalues, where the common dips occur from the $[L]$ and $[M]$ matrices. As predicted analytically in (2.25) and (2.27), spurious eigenvalues, $k_{\mathrm{s}}$, are imbedded in the $[U]$ matrix $\left(k_{\mathrm{s}}=4.82,\left\langle J_{0}^{1}=2.4048\right\rangle\right),[L]$ matrix $\left(k_{\mathrm{s}}=0.30,\left\langle J_{0}^{\prime 1}=0\right\rangle, k_{\mathrm{s}}=3.70\right.$, $\left.\left\langle J_{1}^{\prime 1}=1.84118\right\rangle\right),[T]$ matrix $\left(k_{\mathrm{s}}=4.82,\left\langle J_{0}^{1}=2.4048\right\rangle\right)$ and $[M]$ matrix $\left(k_{\mathrm{s}}=0\right.$, $\left.\left\langle J_{0}^{\prime 1}=0\right\rangle, k_{\mathrm{s}}=3.70,\left\langle J_{1}^{\prime 1}=1.84118\right\rangle\right)$, where $J_{n}^{i}$ and $J_{n}^{\prime i}$ are the $i$ th zeros for the $n$ th-order Bessel function $\left(J_{n}(\cdot)\right)$ and the derivative of the Bessel function $\left(J_{n}^{\prime}(\cdot)\right)$. Since the inner radius $r_{1}$ is $0.5 \mathrm{~m}$, the numerical data and the exact solution in the middle bracket differs by half theoretically. For the $[T]$ matrix, the spurious eigenvalue $\left(k_{\mathrm{s}}=4.82,\left\langle J_{0}^{1}=2.4048\right\rangle\right)$ happens to be almost equal to the true eigenvalue $\left(k_{\mathrm{t}}=4.82\right)$ for the true eigenequation $J_{8}^{\prime}\left(k r_{1}\right) Y_{8}^{\prime}\left(k r_{2}\right)-J_{8}^{\prime}\left(k r_{2}\right) Y_{8}^{\prime}\left(k r_{1}\right)=0$ of the Neumann boundary condition shown in table 1. To sort out the true eigenvalues for the Dirichlet and Neumann problems, the SVD techniques using the updating terms

$$
\left[\begin{array}{l}
U \\
L
\end{array}\right] \text { and } \quad\left[\begin{array}{l}
T \\
M
\end{array}\right]
$$

in (7.21) and (7.24), respectively, were adopted. All the true eigenvalues were extracted out, as can be seen in figure 6. It is interesting to note that, for the Dirichlet problem (see figure $6 a$ ), they are extracted from the updating term

$$
\left[\begin{array}{l}
U \\
L
\end{array}\right]
$$

whereas for the Neumann problem (see figure $6 b$ ) they are from the updating term

$$
\left[\begin{array}{l}
T \\
M
\end{array}\right] \text {. }
$$




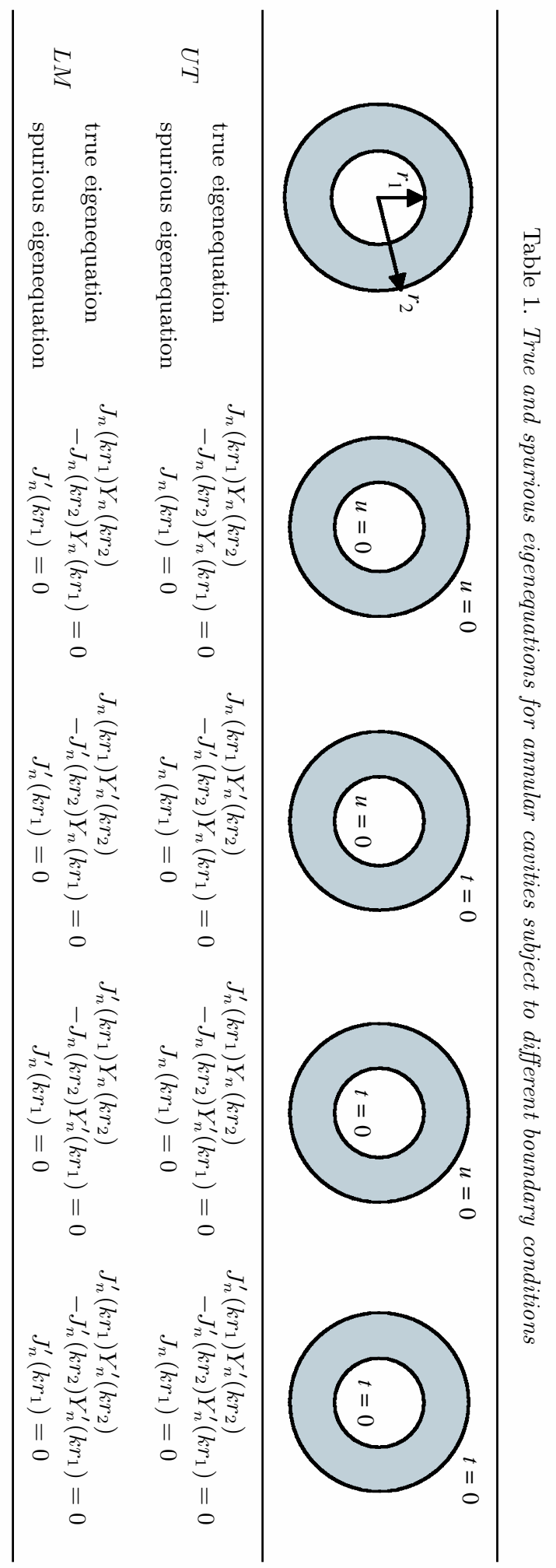



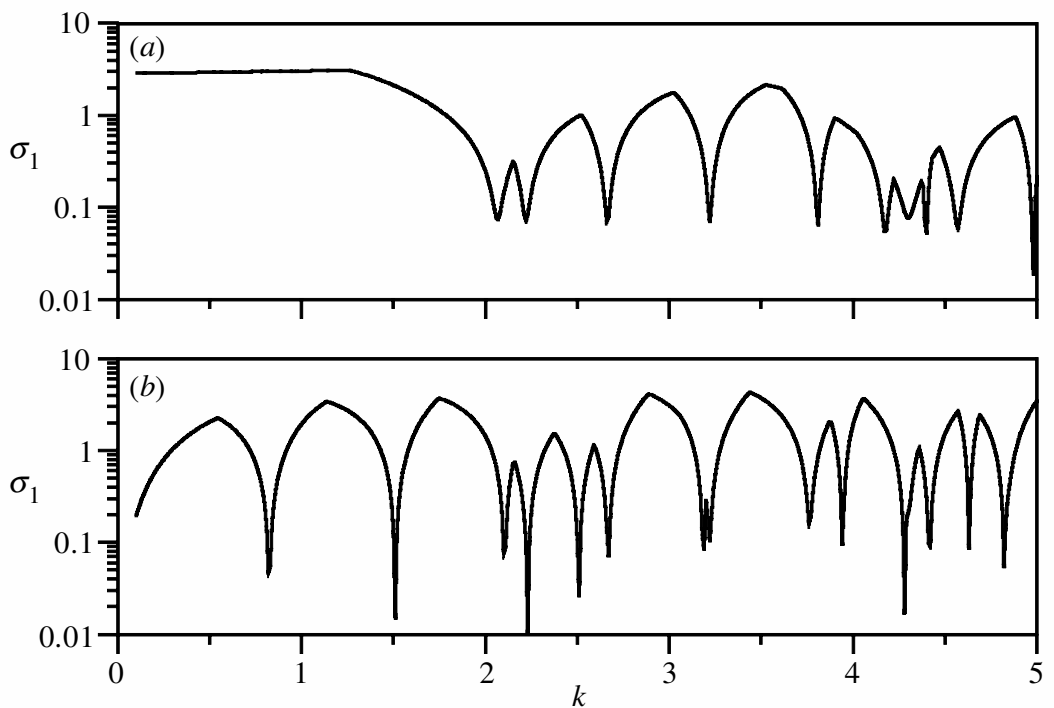

Figure 6. Detection of the true eigenvalues by using the SVD-updating terms.

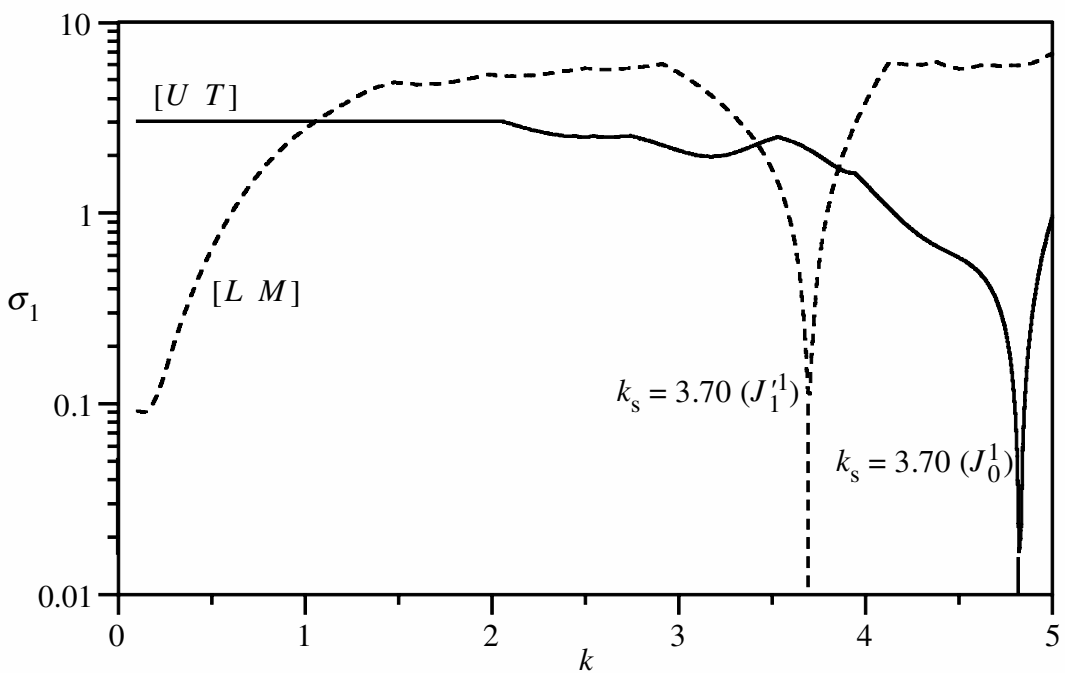

Figure 7. Detection of the spurious eigenvalues by using the SVD-updating documents $[U T]$ and $\left[\begin{array}{ll}L & M\end{array}\right]$. Solid and dashed lines denote the minimum singular value $\sigma_{1}$ for the SVD-updating documents $[U T]$ and $[L M]$, respectively.

It is found that the contamination of all the spurious eigenvalues in the $[U],[L],[T]$ and $[M]$ matrices is suppressed.

To detect the spurious eigenvalues for the $U T$ and $L M$ formulations, the SVD techniques using the updating documents $\left[\begin{array}{ll}U & T\end{array}\right]$ in (7.15) and $\left[\begin{array}{ll}L & M\end{array}\right]$ in (7.16) were adopted, and all the spurious eigenvalues $\left(J_{n}\left(k r_{1}\right)=0\right.$ and $\left.J_{n}^{\prime}\left(k r_{1}\right)=0\right)$ were sorted out and all the true eigenvalues were suppressed, as can be seen in figure 7. After adding a CHIEF point, the spurious eigenvalues of multiplicity one were filtered out, as shown in figure 8 (cf. figure 5). The spurious eigenvalues of 

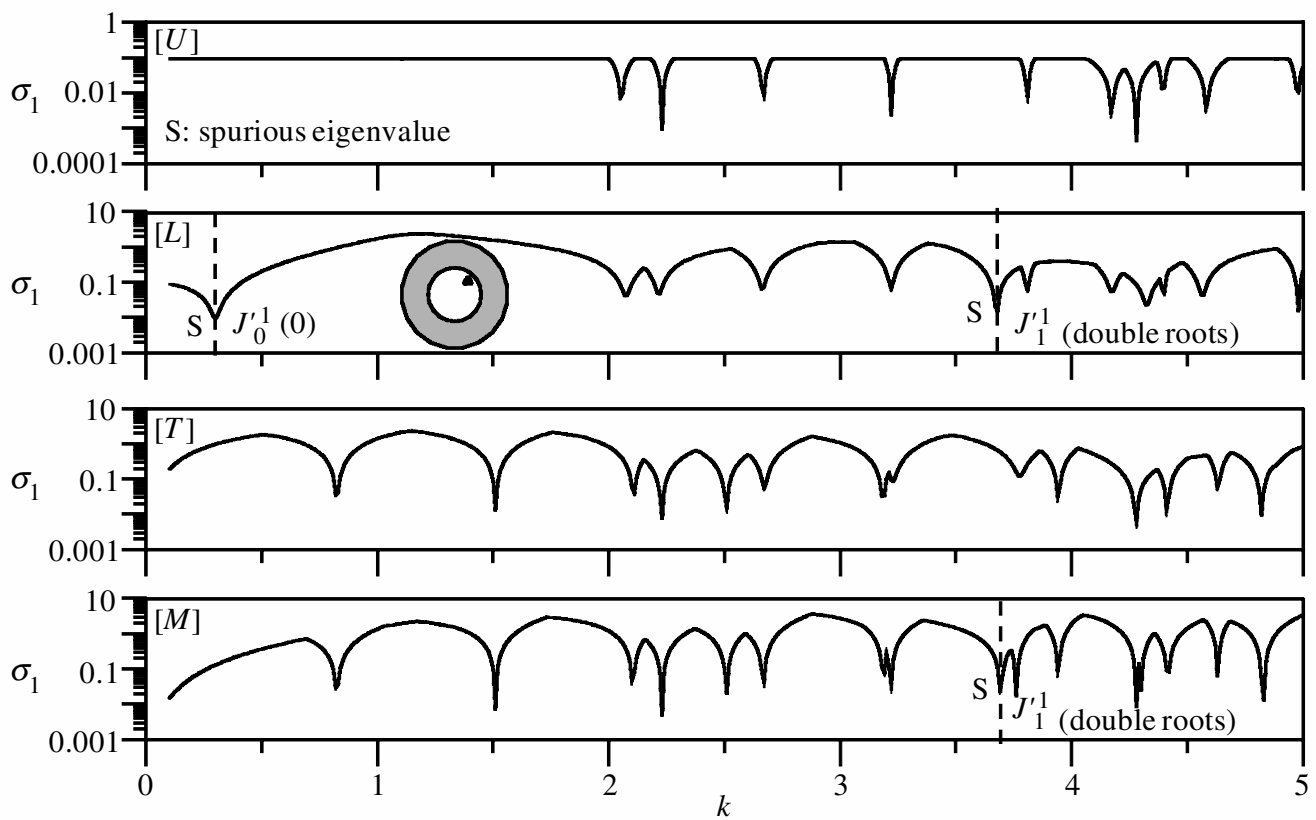

Figure 8. The minimum singular values $\left(\sigma_{1}\right)$ versus $k$ for the $[U],[L],[T]$ and $[M]$ matrices by adding a valid CHIEF point.
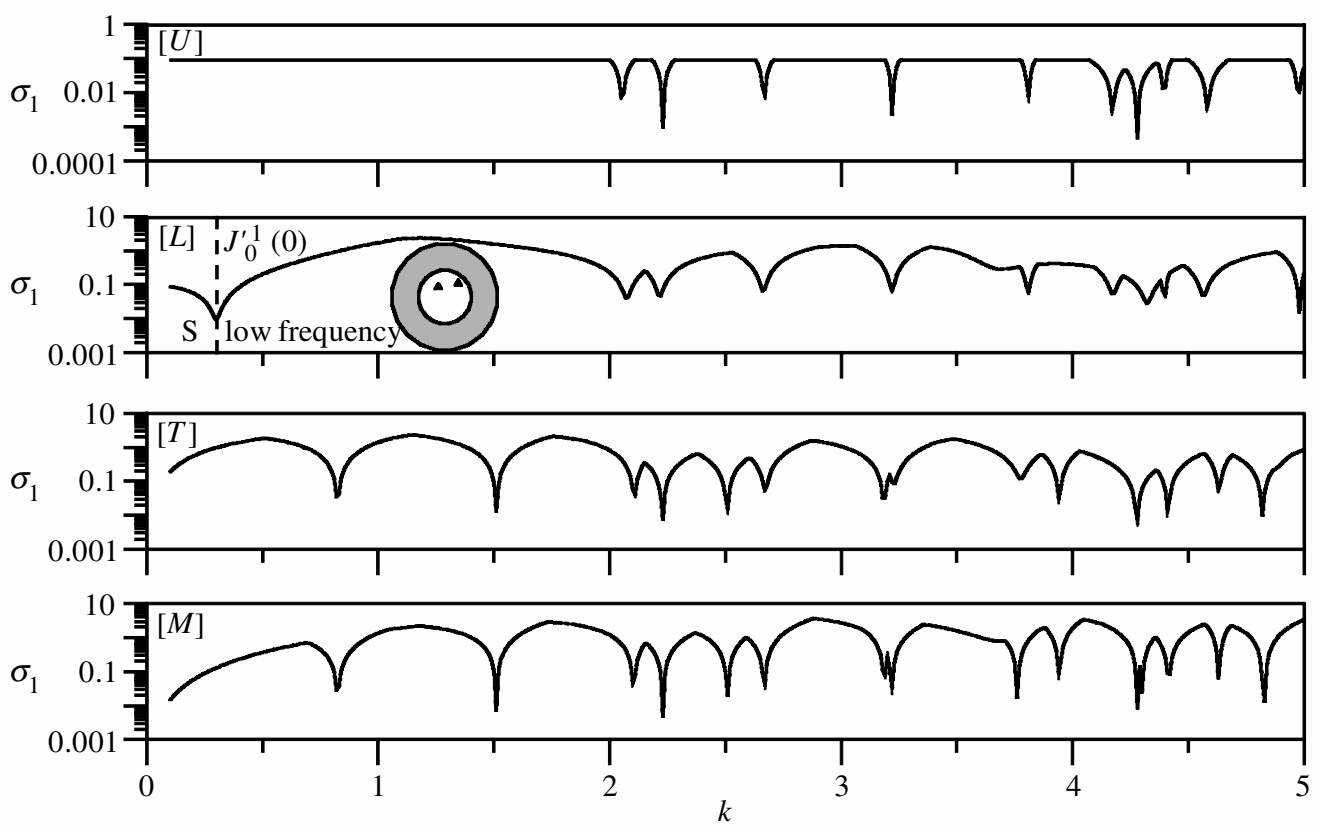

Figure 9. The minimum singular values $\left(\sigma_{1}\right)$ versus $k$ for the $[U],[L],[T]$ and $[M]$ matrices by adding two valid CHIEF points. 

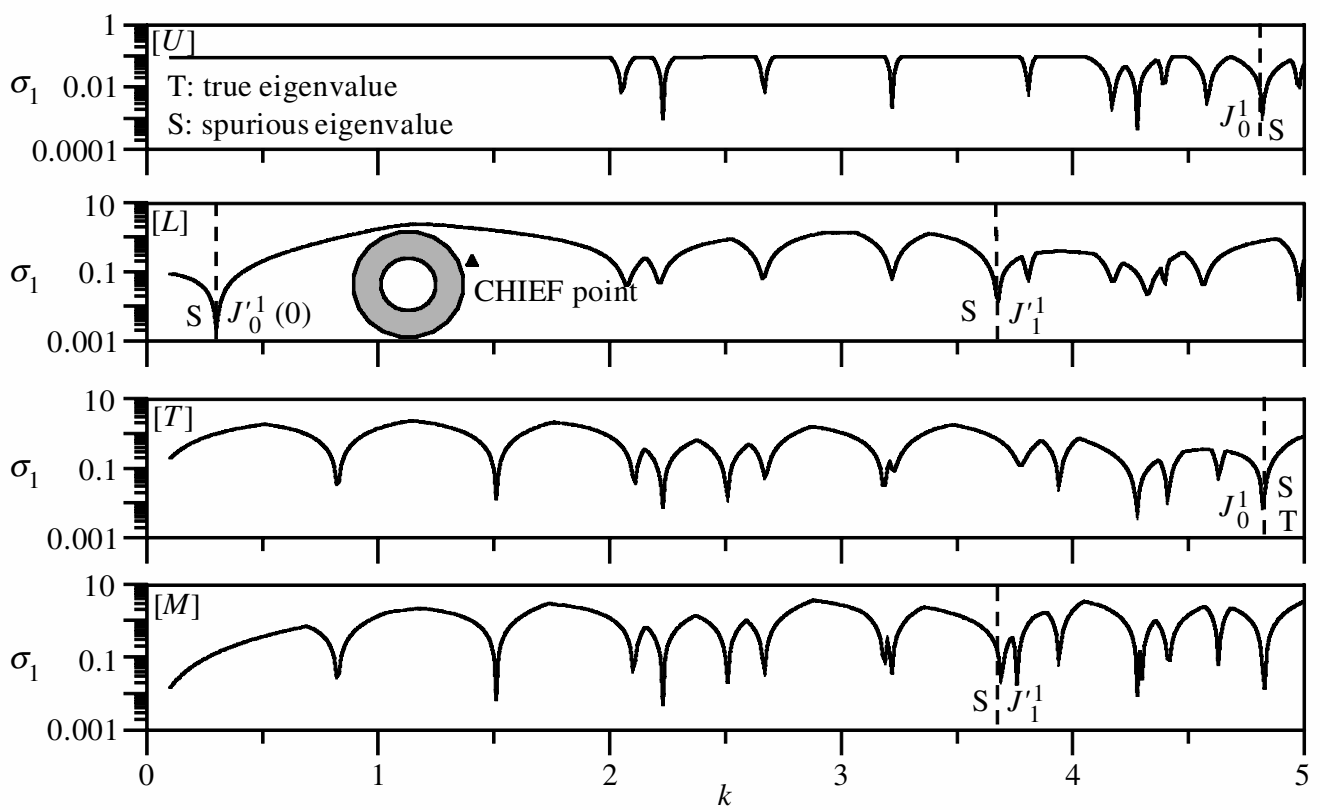

Figure 10. The minimum singular values $\left(\sigma_{1}\right)$ versus $k$ for the $[U],[L],[T]$ and $[M]$ matrices by adding an invalid exterior CHIEF point.
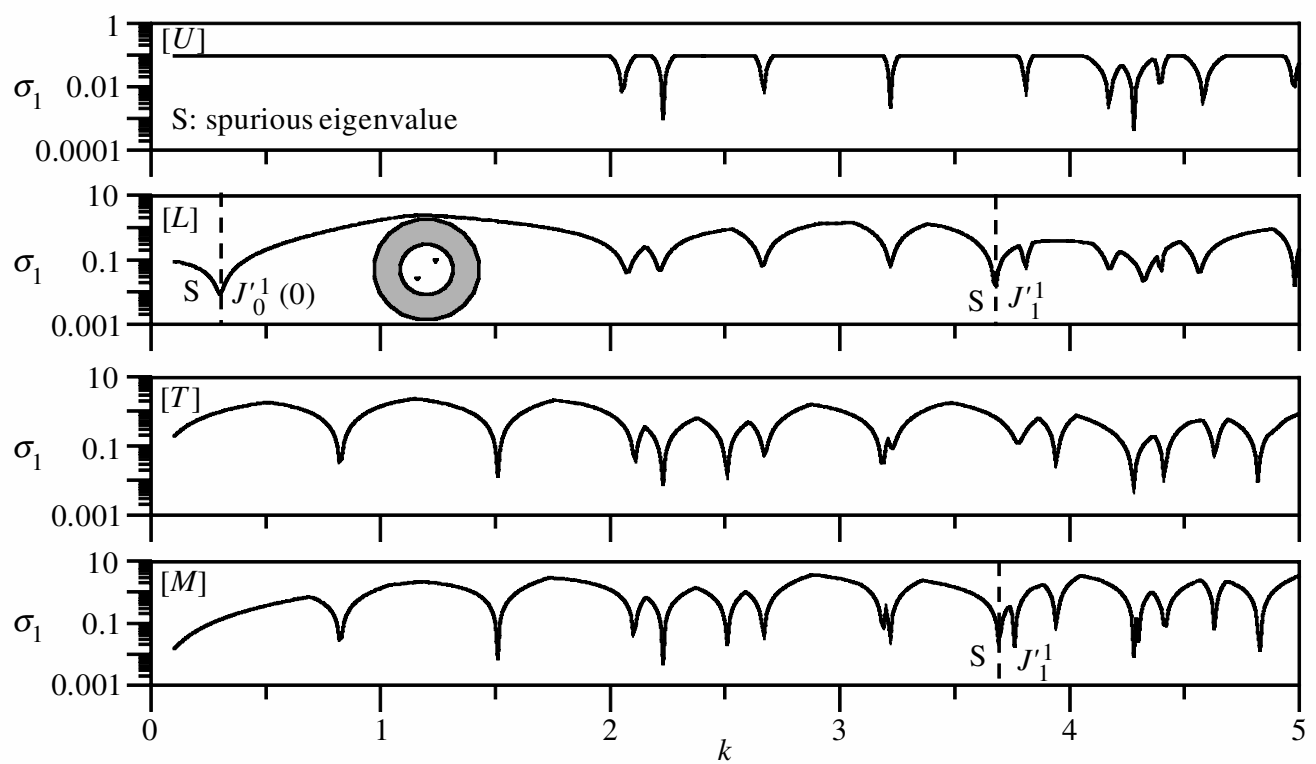

Figure 11. The minimum singular values $\left(\sigma_{1}\right)$ versus $k$ for the $[U],[L],[T]$ and $[M]$ matrices by adding two failure CHIEF points with an intersection angle of $\sin n\left(\phi_{1}-\phi_{3}\right)=0$.

multiplicity two (double roots) still existed as in figure 8. To suppress the spurious eigenvalues of multiplicity two, two CHIEF points were required to filter out all the spurious eigenvalues, as shown in figure 9. To demonstrate the failure in selecting points outside the outer boundary, figure 10, when compared with figure 5, shows 


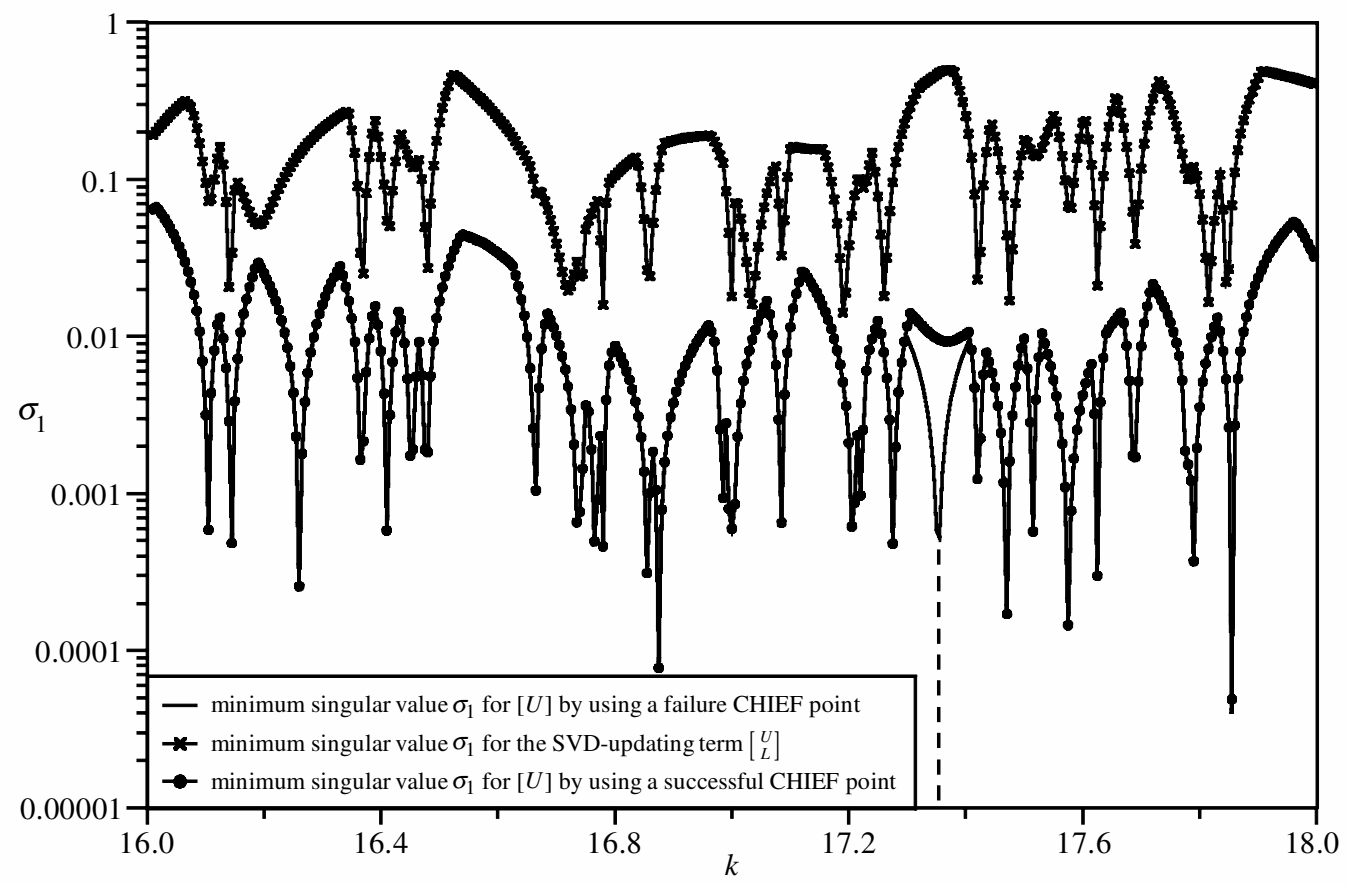

Figure 12. The minimum singular values $\left(\sigma_{1}\right)$ versus $k$ for the $[U]$ matrix by adding an interior failure CHIEF point; $k=17.355\left(J_{0}^{3} / r_{1}=17.3074\right)$.

that spurious eigenvalues still appeared and were not suppressed by imposing the one CHIEF point. In case of two CHIEF points with the intersection angle that satisfies (6.6), the spurious eigenvalues of multiplicity two could not be suppressed, as shown in figure 11. Also, one interior CHIEF point $(\rho, \phi)$ may fail to filter out the spurious eigenvalue, $k_{\mathrm{s}}^{m}$, once $\rho=J_{0}^{p} / k_{\mathrm{s}}^{m}$ or $\rho=J_{0}^{\prime p} / k_{\mathrm{s}}^{m}$, where $k_{\mathrm{s}}^{m}$ satisfies $k_{\mathrm{s}}^{m} r_{1}=J_{0}^{m}$ or $k_{\mathrm{s}}^{m} r_{1}=J_{0}^{\prime m}$, respectively. Since the valid CHIEF point is inside the inner boundary $\left(\rho<r_{1}\right)$, the integer value of $m$ must be larger than $p$. Figure 12 shows that the CHIEF point $(\rho=0.3189 \mathrm{~m}, p=1)$ failed to filter out the spurious eigenvalue $k_{\mathrm{s}}^{3}=17.3074$ for the $[U]$ matrix.

\section{(ii) Null-field BEMs}

According to the preceding discussion, we find that the spurious eigenvalues calculated by the null-field BEMs depend on the inner collocation radius $\rho_{1}$. The results of the different $\rho_{1}$ are shown in figure $13\left(\rho_{1}=0.45 \mathrm{~m}, \rho_{2}=2.2 \mathrm{~m}\right)$ and figure 14 $\left(\rho_{1}=0.4 \mathrm{~m}, \rho_{2}=2.2 \mathrm{~m}\right.$ ). Comparing figure 5 with figure 13 , the spurious eigenvalues shift from $k_{\mathrm{s}}=J_{0}^{1} / r_{1}=2.4048 / 0.5$ to $k_{\mathrm{s}}=J_{0}^{1} / \rho_{1}=2.4048 / 0.45$ for both the $[U]$ and $[T]$ matrices and from $k_{\mathrm{s}}=J_{1}^{\prime 1} / r_{1}=1.84118 / 0.5$ to $k_{\mathrm{s}}=J_{1}^{\prime 1} / \rho_{1}=1.84118 / 0.45$ for both the $[L]$ and $[M]$ matrices. By changing the collocation radii in the null-field equation from $\left(\rho_{1}=0.45 \mathrm{~m}, \rho_{2}=2.2 \mathrm{~m}\right)$ to $\left(\rho_{1}=0.4 \mathrm{~m}, \rho_{2}=2.2 \mathrm{~m}\right)$, the sensitivity analysis demonstrated that the spurious eigenvalues shift right again by a factor of $0.45 / 0.4$ in a similar way as shown in figure 13 . 


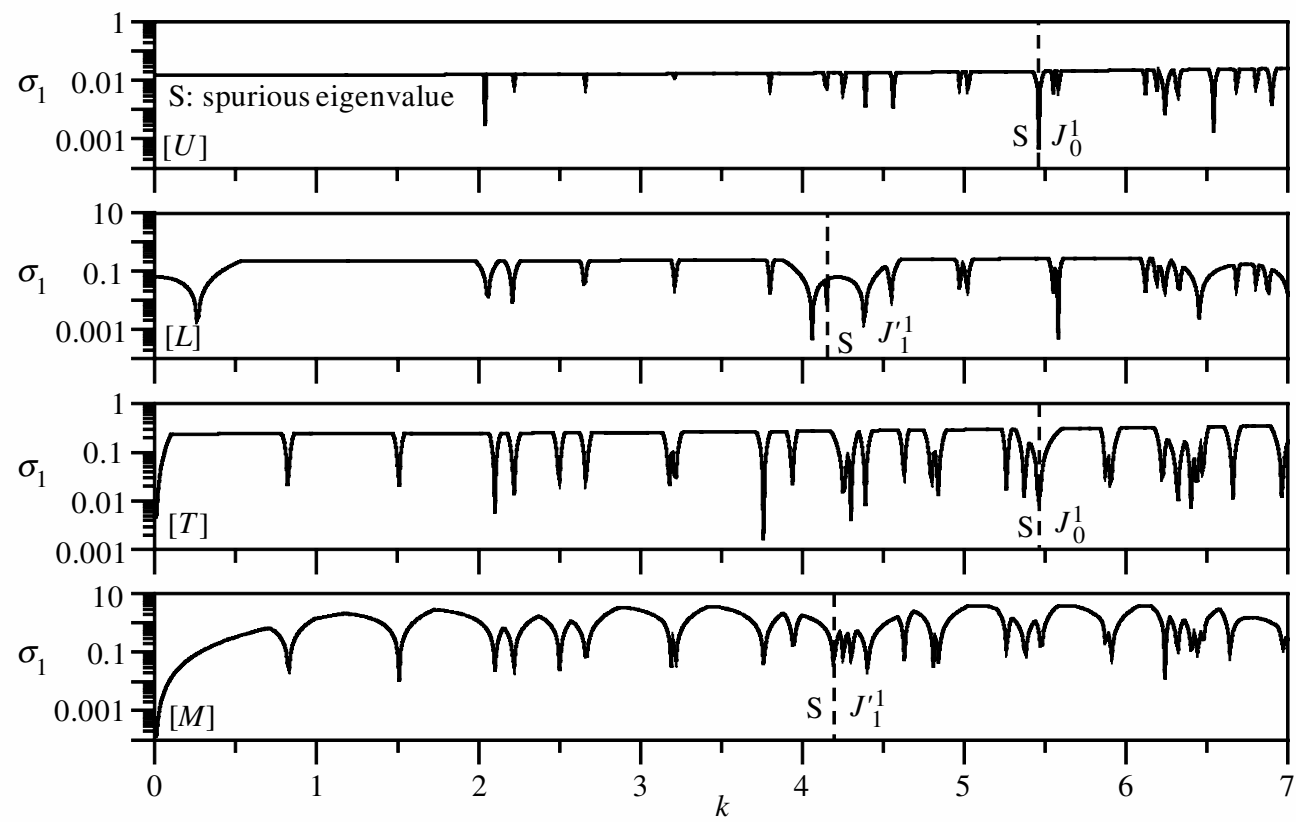

Figure 13. The minimum singular values $\left(\sigma_{1}\right)$ versus $k$ for the $[U],[L],[T]$ and $[M]$ matrices by using the null-field formulation $\left(\rho_{1}=0.45 \mathrm{~m}, \rho_{2}=2.2 \mathrm{~m}\right)$.
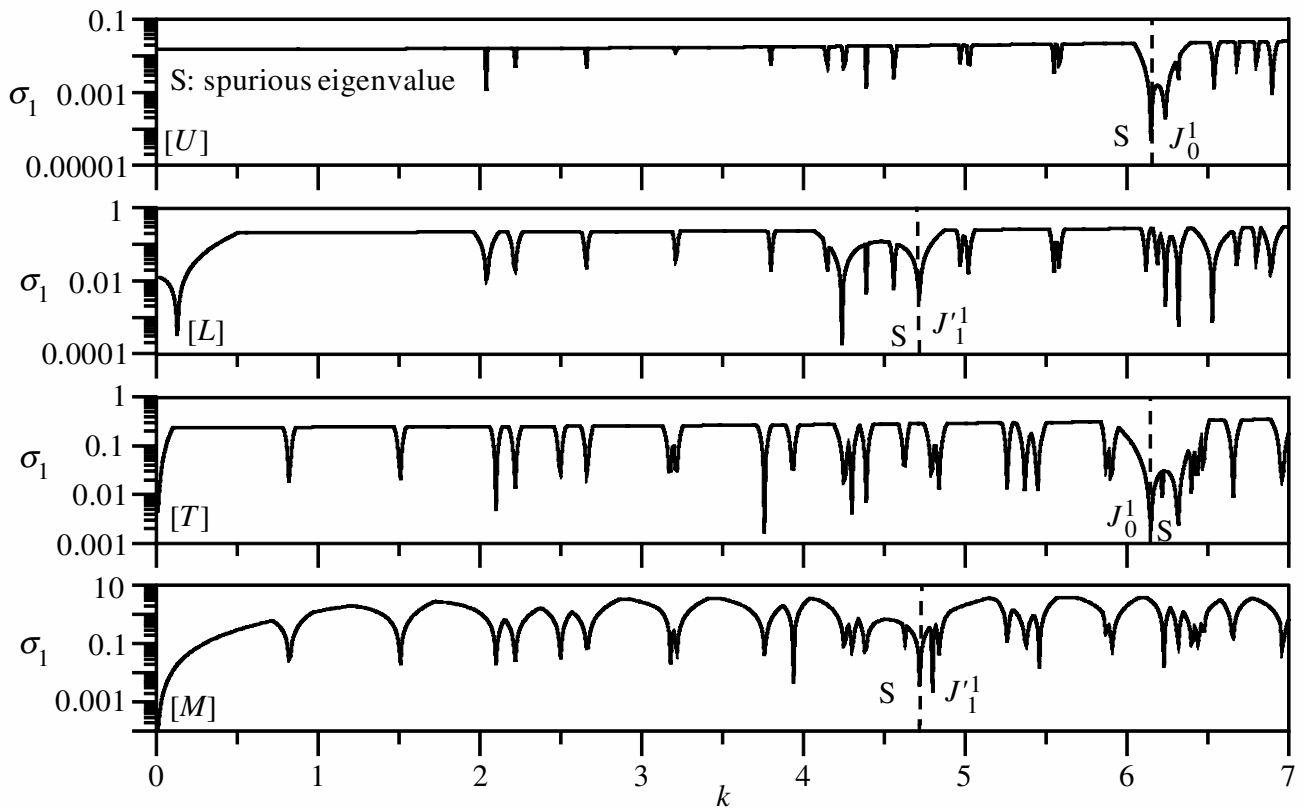

Figure 14. The minimum singular values $\left(\sigma_{1}\right)$ versus $k$ for the $[U],[L],[T]$ and $[M]$ matrices by using the null-field formulation $\left(\rho_{1}=0.4 \mathrm{~m}, \rho_{2}=2.2 \mathrm{~m}\right)$. 
Table 2. The first five eigenvalues of the Helmholtz eigenproblem for the annular cavity using different methods

\begin{tabular}{lcccccc}
\hline method & \multicolumn{5}{c}{ eigenvalue } \\
\cline { 3 - 6 } & $\overbrace{k_{1}}$ & $k_{2}$ & $k_{3}$ & $k_{4}$ & $k_{5}$ \\
\hline FEM $^{\mathrm{a}}$ (ABAQUS) & 2.03 & 2.20 & 2.62 & 3.15 & 3.71 \\
BEM $^{\mathrm{a}}$ (Burton \& Miller) & 2.06 & 2.23 & 2.67 & 3.22 & 3.81 \\
BEM $^{\mathrm{b}}$ (CHIEF) & 2.05 & 2.23 & 2.67 & 3.22 & 3.81 \\
BEM $^{\mathrm{b}}$ (null-field) & 2.04 & 2.20 & 2.65 & 3.21 & 3.80 \\
BEM $^{\mathrm{b}}$ (fictitious) & 2.04 & 2.21 & 2.66 & 3.21 & 3.80 \\
analytical solution $^{\mathrm{b}}$ & 2.05 & 2.23 & 2.66 & 3.21 & 3.80 \\
\hline
\end{tabular}

${ }^{\mathrm{a}}$ Data from Chen et al. $(2001 c) .{ }^{\mathrm{b}}$ Present paper.

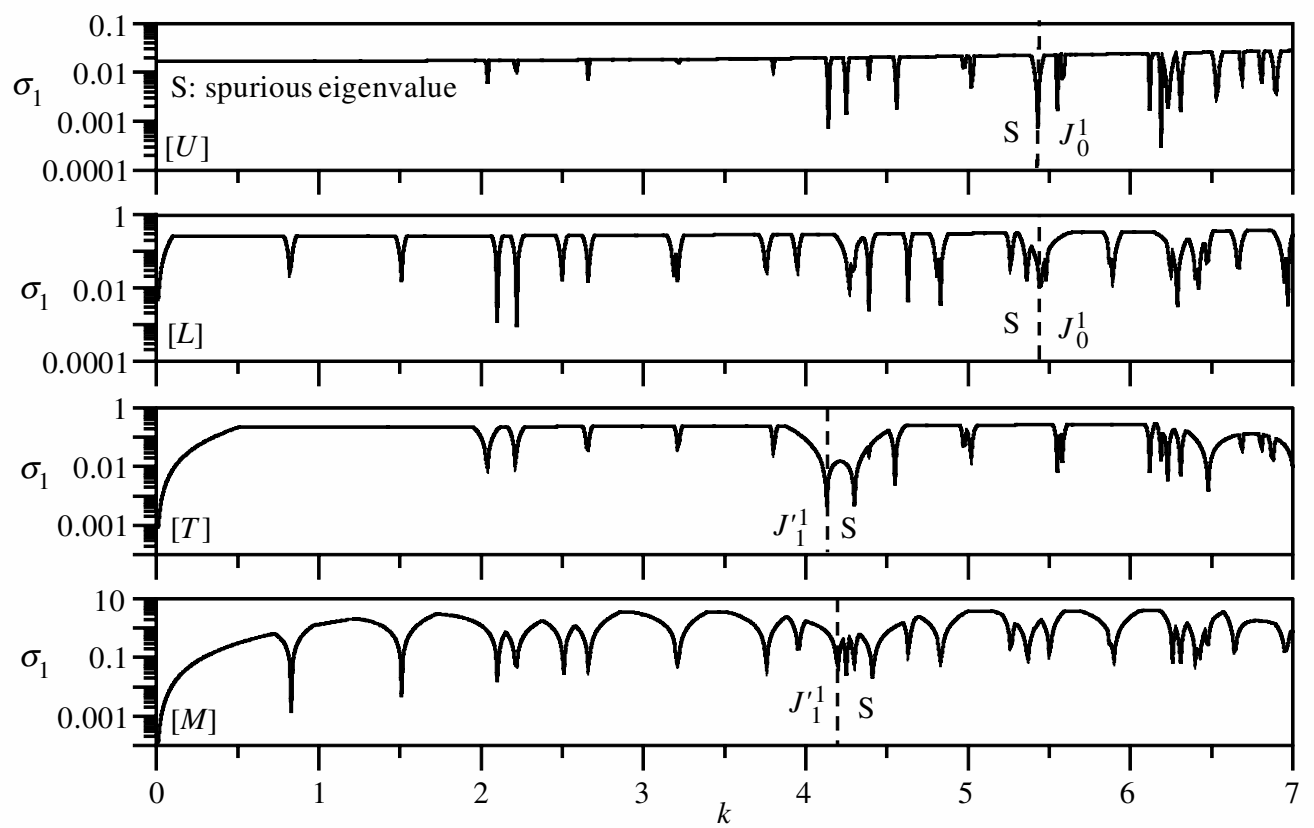

Figure 15. The minimum singular values $\left(\sigma_{1}\right)$ versus $k$ for the $[U],[L],[T]$ and $[M]$ matrices by using the fictitious BEM $\left(r_{1}^{\prime}=0.45 \mathrm{~m}, r_{2}^{\prime}=2.2 \mathrm{~m}\right)$.

(iii) Fictitious BEMs

In the fictitious BEM, the spurious eigenvalues are dominated by the location of the inner fictitious boundary. To demonstrate how the location of the inner fictitious boundary dominates the spurious eigenvalues in (5.11) and (5.22), the fictitious BEMs with $r_{1}^{\prime}=0.4 \mathrm{~m}$ and $r_{2}^{\prime}=2.2 \mathrm{~m}$ were employed to solve the annular case, as shown in figure 15. It is found that the spurious eigenvalues shift to the right by a factor of $\left(r_{1} / r_{1}^{\prime}\right)$, when comparing with figure 5 using the direct singular and hypersingular BEMs. This finding matches the results of the null-field BEMs. By changing the location of inner fictitious boundary from $r_{1}^{\prime}=0.45 \mathrm{~m}$ to $0.4 \mathrm{~m}$, figure 16 shows that spurious eigenvalues shift to the right again by a factor of $(0.45 / 0.4)$ in com- 

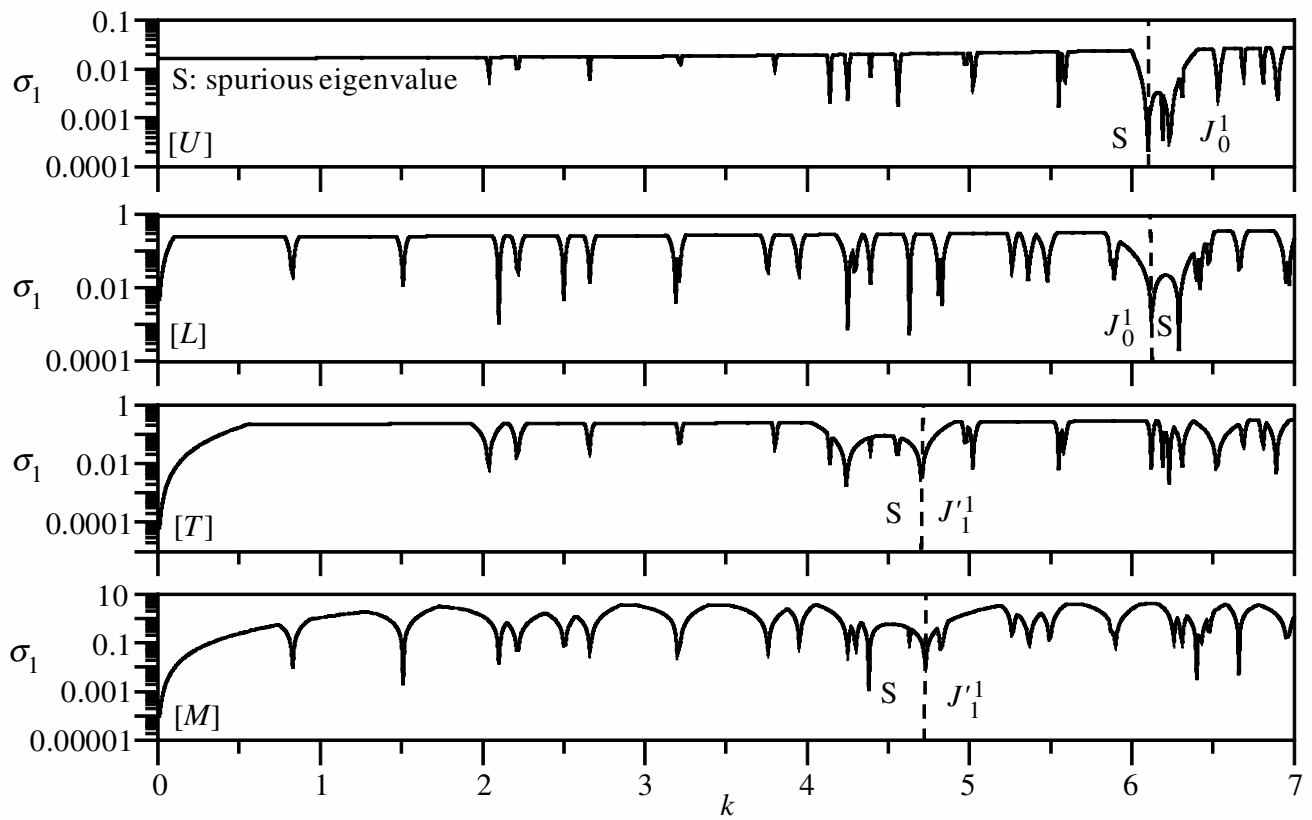

Figure 16. The minimum singular values $\left(\sigma_{1}\right)$ versus $k$ for the $[U],[L],[T]$ and $[M]$ matrices by using the fictitious BEM $\left(r_{1}^{\prime}=0.4 \mathrm{~m}, r_{2}^{\prime}=2.2 \mathrm{~m}\right)$.
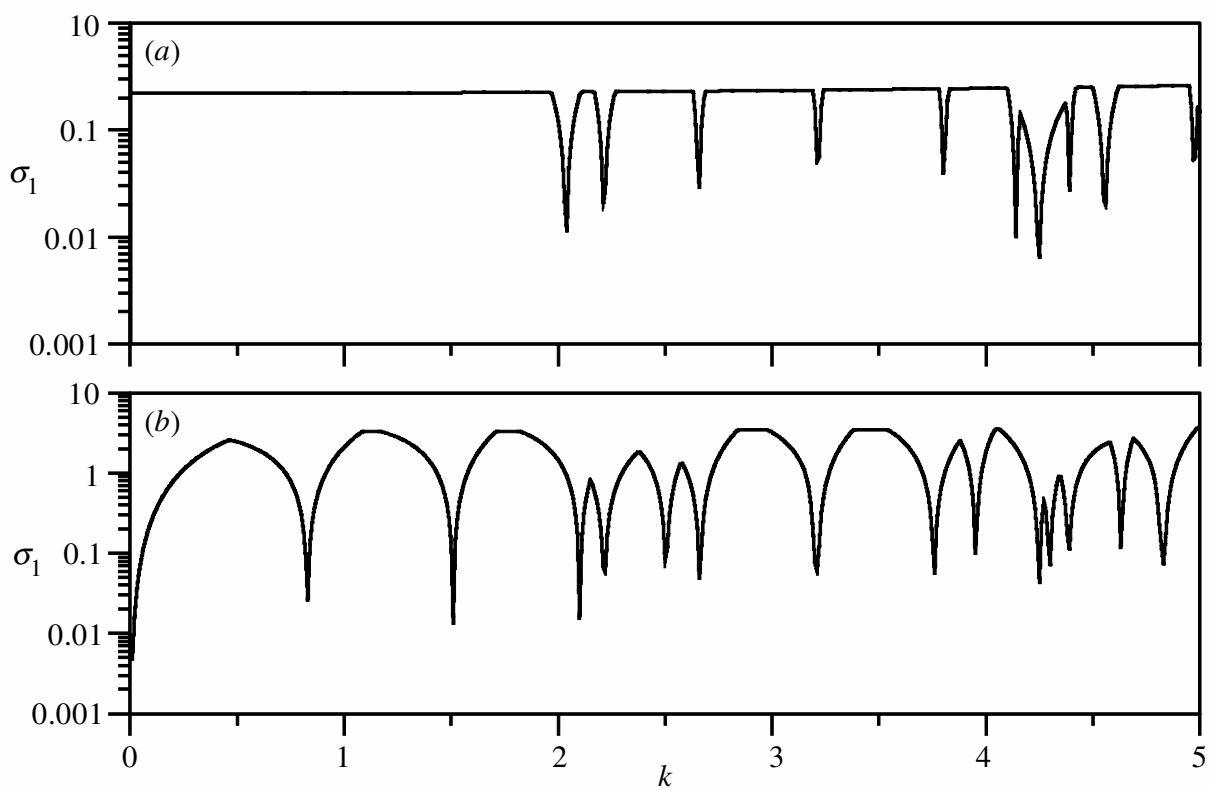

Figure 17. Detection of the true eigenvalues by using the SVD-updating documents $(a)[U T]$ (Dirichlet boundary condition) and $(b)\left[\begin{array}{ll}L & M\end{array}\right]$ (Neumann boundary condition) for the fictitious BEM. 

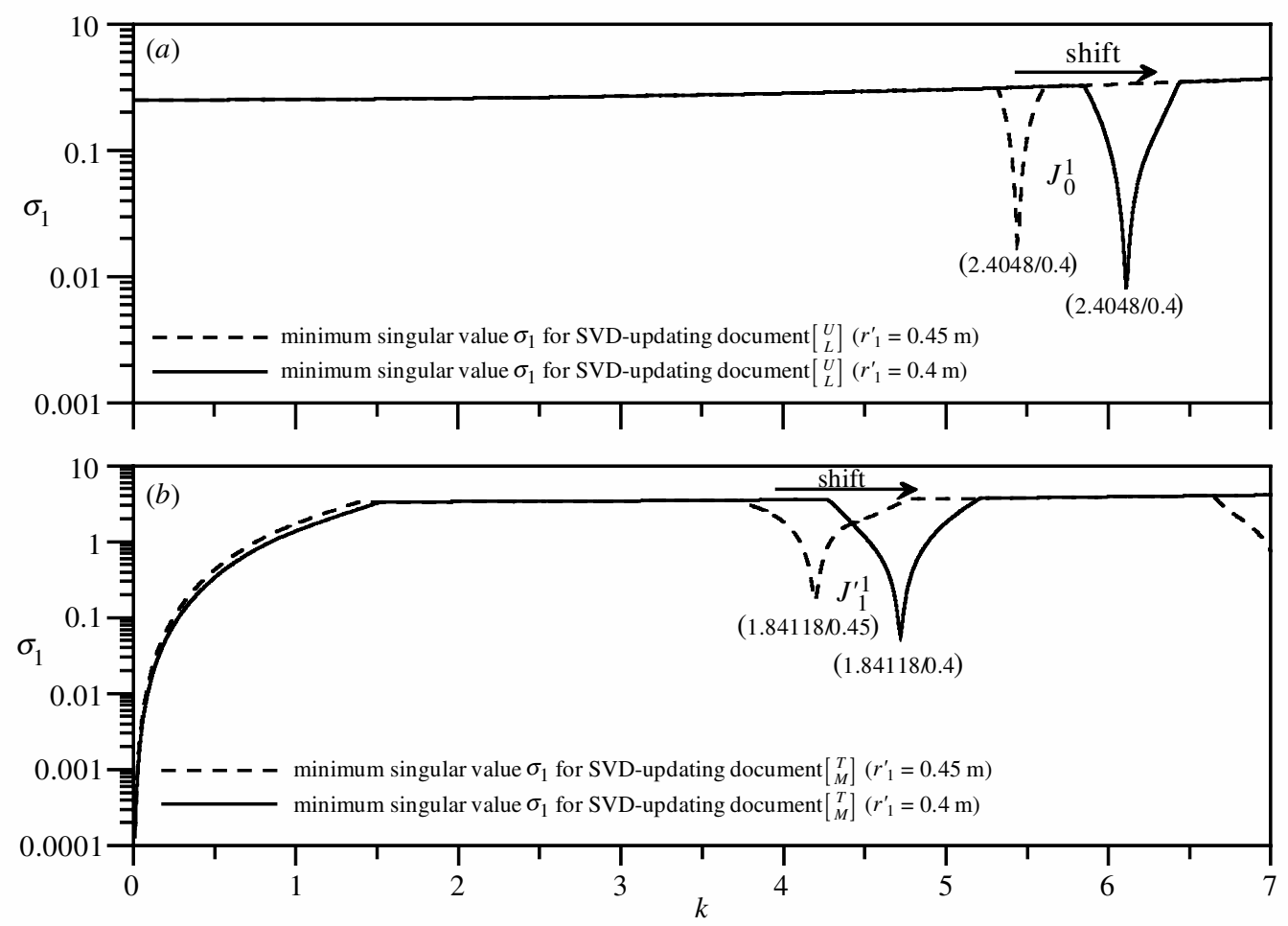

Figure 18. Detection of the spurious eigenvalues by using the SVD-updating documents

$$
\text { (a) } \quad\left[\begin{array}{l}
U \\
L
\end{array}\right] \text { and }(b) \quad\left[\begin{array}{l}
T \\
M
\end{array}\right]
$$

for the fictitious BEM.

parison with those of figure 15. To sort out the true eigenvalues for the Dirichlet and Neumann problems, the SVD technique, using the updating documents $\left[\begin{array}{ll}U & T\end{array}\right]$ in (7.38) and $\left[\begin{array}{ll}L & M\end{array}\right]$ were adopted, respectively. All the true eigenvalues we obtained agree well with those of figure 6 , as shown in figure 17 . To summarize, we list the first five eigenvalues by using different formulations in table 2 . In order to detect the spurious eigenvalues by employing the single-layer- and double-layer-potential approaches, the SVD technique using the updating terms

$$
\left[\begin{array}{l}
U \\
L
\end{array}\right] \quad \text { and } \quad\left[\begin{array}{l}
T \\
M
\end{array}\right]
$$

in (7.41) and (7.42), respectively, were adopted. It is found that all the spurious eigenvalues were sorted out and all the true eigenvalues were suppressed, as in figure 18.

\section{(b) Polygonal cavity}

The polygonal cavities with a fixed circular core are shown in figure 19, with the radius $b=0.5 \mathrm{~m}$ of the circular core and $r=1 \mathrm{~m}$, with a differing number of sides. In this problem, Wang (1998) derived the fundamental frequencies of the polygonal cavities for different numbers of sides $(M)$ of the regular polygon. The fundamental 


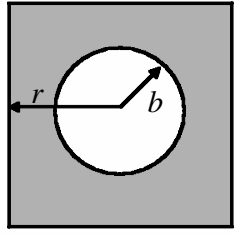

square
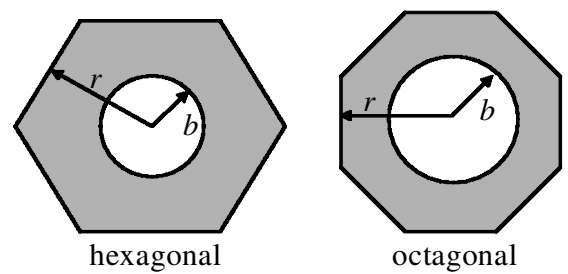

Figure 19. Polygonal acoustic cavities with a fixed circular boundary.

Table 3. The fundamental eigenvalues of the polygonal cavities for different methods

\begin{tabular}{lccc}
\hline & $\begin{array}{c}\text { square } \\
(M=4)\end{array}$ & $\begin{array}{c}\text { hexagonal } \\
(M=6)\end{array}$ & $\begin{array}{c}\text { octagonal } \\
(M=8)\end{array}$ \\
\hline analytical solution $^{\mathrm{a}}$ & 5.09 & 5.76 & 5.97 \\
direct BEM $^{\mathrm{b}}$ & 5.07 & 5.74 & 5.94 \\
CHIEF $^{\mathrm{b}}$ & 5.08 & 5.75 & 5.96 \\
null-field formulation $^{\mathrm{b}}$ & 5.08 & 5.74 & 5.93 \\
fictitious BEM $^{\mathrm{b}}$ & 5.08 & 5.73 & 5.96 \\
\hline
\end{tabular}

${ }^{\mathrm{a}}$ Data from Wang (1998). ${ }^{\mathrm{b}}$ Present paper.

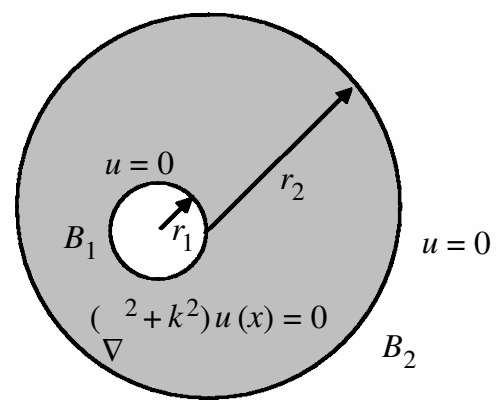

Figure 20. Figure sketch for an eccentric cavity.

frequencies of the polygonal cavities, by using different methods including Wang's result and the methods that we proposed in the present paper, are shown in table 3. The results of the present ones matched well with Wang's result. Since the inner boundary of the polygonal cavity is also a circle, the spurious eigenvalues appear at the same locations as those of the annular case.

\section{(c) Eccentric cavity}

The eccentric cavity is shown in figure 20 , where the inner and outer radii are $r_{1}=$ $0.5 \mathrm{~m}$ and $r_{2}=2 \mathrm{~m}$, respectively. We employed the different methods to determine the first ten eigenvalues, as shown in table 4, in which we also list the results of Chen \& Zhou (1993) and FEM (ABAQUS). The results of the singular and hypersingular BEMs, the CHIEF method, the null-field formulations and the fictitious BEMs agree well with those of Chen \& Zhou (1993) and FEM (ABAQUS). Similarly, the inner circular boundary dominates the spurious eigenvalues, although it is eccentric. The spurious eigenvalues are identical to those of the annular cavity. 
Table 4. The first ten eigenvalues of the Helmholtz eigenproblem for the eccentric case using different methods

\begin{tabular}{lcccccccccc}
\hline & 1 & 2 & 3 & 4 & 5 & 6 & 7 & 8 & 9 & 10 \\
\hline FEM $^{\mathrm{a}}$ (ABAQUS) & 1.73 & 2.13 & 2.45 & 2.76 & 2.95 & 3.30 & 3.34 & 3.36 & 3.83 & 3.84 \\
Chen \& Zhou (1993) & 1.75 & 2.14 & 2.47 & 2.78 & 2.97 & 3.33 & 3.37 & 3.38 & 3.85 & 3.87 \\
Burton \& Miller (1971) & 1.74 & 2.14 & 2.47 & 2.78 & 2.97 & 3.33 & 3.37 & 3.39 & 3.87 & 3.87 \\
direct BEM $^{\mathrm{b}}$ & 1.73 & 2.13 & 2.46 & 2.77 & 2.95 & 3.31 & 3.35 & 3.37 & 3.85 & 3.85 \\
CHIEF $^{\mathrm{b}}$ & 1.73 & 2.13 & 2.46 & 2.76 & 2.95 & 3.31 & 3.35 & 3.37 & 3.84 & 3.86 \\
null-field formulation $^{\mathrm{b}}$ & 1.73 & 2.12 & 2.45 & 2.76 & 2.95 & 3.31 & 3.34 & 3.36 & 3.83 & 3.85 \\
fictitious BEM $^{\mathrm{b}}$ & 1.73 & 2.13 & 2.45 & 2.76 & 2.95 & 3.31 & 3.35 & 3.35 & 3.86 & 3.86 \\
\hline
\end{tabular}

${ }^{\mathrm{a}}$ Data from Chen et al. $(2001 c) .{ }^{\mathrm{b}}$ Present paper.

\section{Concluding remarks}

In this paper, we have verified that the spurious eigensolutions depend on the inner boundary of a multiply connected problem for both the continuous and the discrete formulations. The spurious and true eigenmodes for the annular domain were analytically derived by using the degenerate kernel and the Fourier series expansion. Singularity-free methods, such as the null-field formulation and the fictitious BEM, also suffer from the problem of spurious eigenvalues that depend on the locations of the collocation points and the fictitious boundaries, respectively. Hence the CHIEF method and the SVD-updating techniques were employed to overcome the spurious eigensolutions. The true and spurious modes are found to be the right and left unitary vectors, respectively, of the four influence matrices in the dual BEM. The positions of the CHIEF points should be chosen inside the inner boundary and must avoid locating at the point such that $J_{n}(k \rho)=0$ and $\sin n\left(\phi_{1}-\phi_{3}\right)=0$. Also, the criteria of failure CHIEF points for the null-field formulation and the fictitious BEM are similar to that of singular and hypersingular BEMs. For singular and hypersingular BEMs, the null-field approach and the fictitious BEM, the spurious and true eigenvalues were detected successfully by using the SVD-updating techniques and the Fredholm alternative theorem. In the numerical experiments, three cases showed that the null-field formulation and the fictitious BEM can obtain accurate results as well as the singular and hypersingular BEMs do. But the locations of the collocation points of the null-field formulation and fictitious boundaries of the fictitious BEM must be chosen carefully, so as to avoid not only the spurious eigenvalue, but also the ill-posed behaviour. In addition, the efficiency of the CHIEF method is better than that of the Burton-Miller method since not only is hypersingularity avoided, but also computation effort is saved. The cases of polygonal and eccentric cavities also tend to indicate the validity of the proposed methodologies for the problems with general boundaries.

Financial support from the National Science Council, under Grant no. NSC-91-2211-E-019-009, to the National Taiwan Ocean University is gratefully acknowledged.

\section{References}

Achenbach, J. D., Kechter, G. E. \& Xu, Y.-L. 1988 Off-boundary approach to the boundary element method. Computat. Meth. Appl. Mech. Engng 70, 191-201. 
Berry, M. W., Drmac, Z. \& Jessup, E. R. 1999 Matrices, vector spaces, and information retrieval. SIAM Rev. 41, 335-362.

Burton, A. J. \& Miller, G. F. 1971 The application of integral equation methods to numerical solution of some exterior boundary value problems. Proc. R. Soc. Lond. A 323, 201-210.

Chen, J. T. 1998 On the fictitious frequencies using dual series representation. Mech. Res. Commun. 25, 529-534.

Chen, J. T. \& Chen, K. H. 1998 Dual integral formulation for determining the acoustic modes of a two-dimensional cavity with a degenerate boundary. Engng Analysis Bound. Elem. 21, 105-116.

Chen, J. T. \& Hong, H.-K. 1999 Review of dual boundary element methods with emphasis on hypersingular integral and divergent series. Appl. Mech. Rev. 52, 17-33.

Chen, J. T. \& Kuo, S. R. 2000 On fictitious frequencies using circulants for radiation problems of a cylinder. Mech. Res. Commun. 27, 49-58.

Chen, G. \& Zhou, J. 1993 Vibration and damping in distributed systems vol. 2. Boca Raton, FL: Chemical Rubber Company.

Chen, J. T., Huang, C. X. \& Wong, F. C. $2000 a$ Determination of spurious eigenvalues and multiplicities of true eigenvalues in the dual multiple reciprocity method using the singular value decomposition technique. J. Sound Vib. 230, 219-230.

Chen, J. T., Kuo, S. R. \& Cheng, Y. C. $2000 b$ On the true and spurious eigensolutions using circulants for real-part dual BEM. In IUTAM/IACM/IABEM Symp. Advanced Mathematical and Computational Mechanics Aspects of Boundary Element Method, Cracow, Poland, pp. 7785. Dordrecht: Kluwer.

Chen, I. L., Chen, J. T., Kuo, S. R. \& Liang, M. T. 2001a A new method for true and spurious eigensolutions of arbitrary cavities using the CHEEF method. J. Acoust. Soc. Am. 109, 982999.

Chen, I. L., Chen, J. T. \& Liang, M. T. $2001 b$ Analytical study and numerical experiments for radiation and scattering problems using the CHIEF method. J. Sound Vib. 248, 809-828.

Chen, J. T., Lin, J. H., Kuo, S. R. \& Chyuan, S. W. 2001c Boundary element analysis for the Helmholtz eigenvalue problems with a multiply connected domain. Proc. R. Soc. Lond. A 457, 2521-2546.

De Mey, G. 1976 Calculation of the Helmholtz equation by an integral equation. Int. J. Numer. Meth. Engng 10, 59-66.

De Mey, G. 1977 A simplified integral equation method for the calculation of the eigenvalues of Helmholtz equation. Int. J. Numer. Meth. Engng 11, 1340-1342.

Golub, G. H. \& Van Loan, C. F. 1989 Matrix computations, 2nd edn. Baltimore, MD: Johns Hopkins University Press.

Hong, H.-K. \& Chen, J. T. 1988 Derivations of integral equations of elasticity. J. Engng Mech. ASME 114, 1028-1044.

Hsiao, G. C. \& Wendland, W. L. 2000 Boundary integral methods in low frequency acoustics. J. Chin. Inst. Engrs 23, 369-375.

Hutchinson, J. R. 1985 An alternative BEM formulation applied to membrane vibrations. In Boundary elements VII (ed. C. A. Brebbia \& G. Maier). Springer.

Hutchinson, J. R. 1988 Vibration of plates, in boundary elements X (ed. C. A. Brebbia), vol. 4, pp. 415-430. Springer.

Hutchinson, J. R. 1991 Analysis of plates and shells by boundary collocation. In Boundary elements analysis of plates and shells (ed. D. E. Beskos), pp. 314-368. Springer.

Hwang, J. Y. \& Chang, S. C. 1991 A retracted boundary integral equation for exterior acoustic problem with unique solution for all wavenumbers. J. Acoust. Soc. Am. 90, 1167-1180.

Itagaki, M. \& Brebbia, C. A. 1993 Source iterative multiple reciprocity technique for Helmholtz eigenvalue problems with boundary elements. In Proc. 5th Japan-China Symp. Boundary Element Methods, Current Research in Japan and China, Sapporo, pp. 79-88. Elsevier.

Proc. R. Soc. Lond. A (2003) 
Itagaki, M. \& Brebbia, C. A. 1994 Application of the multiple reciprocity boundary element method to neutron diffusion problems. In The multiple reciprocity boundary element method. Southampton: Computational Mechanics Publications.

Itagaki, M., Nishiyama, S., Tomioka, S., Enoto, T. \& Sahashi, N. 1997 Power iterative multiple reciprocity boundary element method for solving three-dimensional Helmholtz equation Engng Analysis Bound. Elem. 20, 113-121.

Kamiya, N. \& Andoh, E. 1993 A note on multiple reciprocity integral formulation for Helmholtz equation. Commun. Numer. Meth. Engng 9, 9-13.

Kamiya, N., Andoh, E. \& Nogae, K. 1996 A new complex-valued formulation and eigenvalue analysis of the Helmholtz equation by boundary element method. Adv. Engng Software 26, 219-227.

Kitahara, M. 1985 Boundary integral equation methods in eigenvalue problems of elastodynamics and thin plates. Elsevier.

Kuo, S. R., Chen, J. T. \& Huang, C. X. 2000a Analytical study and numerical experiments for true and spurious eigensolutions of a circular cavity using the real-part dual BEM. Int. J. Numer. Meth. Engng 48, 1404-1422.

Kuo, S. R., Yeih, W. \& Wu, Y. C. $2000 b$ Applications of the generalized singular-value decomposition method on the eigenproblem using the incomplete boundary element formulation. $J$. Sound Vib. 235, 813-845.

Martin, P. A. 1980 On the null-field equations for the exterior problems of acoustics. Q. J. Mech. Appl. Math. 27, 386-396.

Nowak, A. J. \& Brebbia, C. A. 1989 The multiple reciprocity method-a new approach for transforming BEM domain integrals to the boundary. Engng Analysis Bound. Elem. 6, 164167.

Nowak, A. J. \& Neves, A. C. (eds) 1994 Multiple reciprocity boundary element method. Southampton: Computational Mechanics Publications.

Partridge, P. W., Brebbia, C. A. \& Wrobel, L. C. 1992 The dual reciprocity boundary element method. Southampton: Computational Mechanics Publications.

Schenck, H. A. 1968 Improved integral formulation for acoustic radiation problem. J. Acoust. Soc. Am. 44, 41-58.

Seybert, A. F. \& Rengarajan, T. K. 1987 The use of CHIEF to obtain unique solutions for acoustic radiation using boundary integral equations. J. Acoust. Soc. Am. 81, 1299-1306.

Tai, G. R. G. \& Shaw, R. P. 1974 Helmholtz equation eigenvalues and eigenmodes for arbitrary domains. J. Acoust. Soc. Am. 56, 796-804.

Wang, C. Y. 1998 On the polygonal membrane with a circular core. J. Sound Vib. 215, 195-199.

As this paper exceeds the maximum length normally permitted, the authors have agreed to contribute to production costs. 